\title{
RNA folding kinetics using Monte Carlo and Gillespie algorithms*
}

\author{
Peter Clote Amir H. Bayegan \\ Department of Biology, Boston College, Chestnut Hill, MA 02467, USA. clote@bc.edu.
}

\begin{abstract}
RNA secondary structure folding kinetics is known to be important for the biological function of certain processes, such as the hok/sok system in E. coli. Although linear algebra provides an exact computational solution of secondary structure folding kinetics with respect to the Turner energy model for tiny $(\approx 20 \mathrm{nt}) \mathrm{RNA}$ sequences, the folding kinetics for larger sequences can only be approximated by binning structures into macrostates in a coarse-grained model, or by repeatedly simulating secondary structure folding with either the Monte Carlo algorithm or the Gillespie algorithm.

Here we investigate the relation between the Monte Carlo algorithm and the Gillespie algorithm. We prove that asymptotically, the expected time for a $K$-step trajectory of the Monte Carlo algorithm is equal to $\langle N\rangle$ times that of the Gillespie algorithm, where $\langle N\rangle$ denotes the Boltzmann expected network degree. If the network is regular (i.e. every node has the same degree), then the mean first passage time (MFPT) computed by the Monte Carlo algorithm is equal to MFPT computed by the Gillespie algorithm multiplied by $\langle N\rangle$; however, this is not true for non-regular networks. In particular, RNA secondary structure folding kinetics, as computed by the Monte Carlo algorithm, is not equal to the folding kinetics, as computed by the Gillespie algorithm, although the mean first passage times are roughly correlated.

Simulation software for RNA secondary structure folding according to the Monte Carlo and Gillespie algorithms is publicly available, as is our software to compute the expected degree of the network of secondary structures of a given RNA sequence - see http://bioinformatics.bc.edu/clote/ RNAexpNumNbors
\end{abstract}

\section{Introduction}

Ever since Anfinsen's pioneering experiment involving the denaturation and renaturation of bovine ribonuclease [3, it has long been held that the native state of a protein is that conformation which has minimum free energy (MFE) among all possible conformations. For that reason, both the Monte Carlo algorithm [34] and the Gillespie algorithm 22] have been used in biopolymer folding studies to estimate kinetic folding time, even when detailed balance does not hold ${ }^{1}$ Since a number of different measures of folding time have been described for RNA secondary structure formation [42, 17, 53, 45, the goal of the current paper is to clarify the relations between these measures, to point out the possibly low correlation between mean first passage time (MFPT) when computed by the Monte Carlo algorithm versus Gillespie algorithm, and to prove an asymptotic result that precisely relates Monte Carlo trajectory time with Gillespie trajectory time. We hope that this clarification will be useful to those users who perform computational experiments to estimate RNA folding kinetics.

\section{Protein folding kinetics using Monte Carlo and/or Gillespie}

For protein to be biologically useful, its amino acid sequence must satisfy two requirements: (1) a thermodynamic requirement that the sequence have a unique, thermodynamically stable native structure, and (2) a kinetic requirement that the denatured polypeptide chain be capable of reaching the native state within

\footnotetext{
*Research supported by National Science Foundation grant DBI-1262439.

${ }^{1}$ Definitions of Monte Carlo algorithm, Gillespie algorithm, detailed balance, mean first passage time, master equation, etc. are given later in the paper.
} 
reasonable time (milliseconds to seconds) under appropriate solution conditions. In [40, 41] a simple Monte Carlo folding experiment was designed for a 27 bead hetero-polymer on a cubic lattice using hydrophobic contact potentials. Defining a sequence to fold rapidly provided its MFPT is sufficiently small, the authors concluded that a necessary and sufficient condition for a sequence to fold rapidly in their model is the existence of a pronounced energy minimum with substantial gap between the energy of the native state and that of the most stable misfolded structure. A succinct summary of the theoretical work in [40, 41] is that the thermodynamic requirement entails the kinetic requirement. In 1, 2] Monte Carlo hetero-polymer folding experiments were performed to show how prebiotic proteins might have evolved to fold rapidly in a primordial soup in order to survive hydrolysis. Hetero-polymer sequences were evolved by applying a Metropolis criterion to the acceptance of pointwise mutations, depending on whether the mutation increased MFPT. One of the conclusions from this work was that the kinetic requirement entails the thermodynamic requirement.

More recently, the notion of Markov state model (MSM) has been introduced to analyze protein folding kinetics by statistical analysis of a number of molecular dynamics (MD) trajectories - see for instance [47, 6, 25, 51, 5, 23. To construct a Markov state model, microstates are first formed by applying a clustering algorithm to conformations taken at fixed time intervals (shapshots) from MD trajectories by using a measure of structural similarity such as RMSD. States of the Markov state model are then defined to be kinetically reachable macrostates constructed from microstates, where transition probabilities are defined by counting the relative number of transitions between macrostates. The kinetics of folding are then analyzed by computing MFPT to reach the macrostate of the MSM which contains the native state. Provided that the number of states is reasonably small, the MFPT can be computed analytically from the fundamental matrix as done in 51. Analytic computations of MFPT are much more efficient and accurate than repeated simulations of the Monte Carlo algorithm - however, both methods are theoretically equivalent. In contrast, in some papers such as [58, instead of computing the MFPT of the Markov state model, transition rates are computed from which the master equation is solved to determine the equilibrium time, i.e. time necessary for molecular equilibrium. Provided that the number of states is reasonably small, it is possible to solve the master equation analytically by spectral decomposition; otherwise, the Gillespie algorithm can be used to stochastically estimate the equilibrium time. In [32, a formula (involving the integral of an appropriate time correlation function) is given to estimate the equilibrium time among unfolded states of a protein having two state kinetics.

\section{RNA folding kinetics using Monte Carlo and/or Gillespie}

In 42, the folding time for an RNA sequence is defined to be the sum of reciprocals of the rate constants $k=k_{0} \cdot \exp \left(-\Delta E^{\ddagger} / R T\right)$ in each step of a folding trajectory, where $k_{0}$ is a rate calibration constant and $\Delta E^{\ddagger}$ is the activation energy of adding or removing a base pair to the current structure, using the Turner free energy model [50]. The resultant folding time differs from MFPT by additionally accounting for the transition rates between trajectory steps. A similar notion of folding time is adopted in [21, where additionally basins of attraction are estimated by assigning a collection of sampled secondary structures to a locally optimal structure by a greedy procedure.

In [45, mean first passage time was estimated by the average number of Monte Carlo steps, taken over 50 runs, to fold the empty secondary structure into the MFE structure. The authors determined that for the selenocysteine (SECIS-1) family RF00031 from the Rfam database [37, the Pearson correlation coefficient is 0.48436 ( $\mathrm{p}$-value $1.8 \cdot 10^{-4}$ ) between the logarithm of MFPT for a given RNA sequence and the standard deviation of the number of base pairs taken over the ensemble of all secondary structures of that sequence. After publication of [45, computational experiments performed with the Gillespie algorithm in place of the Monte Carlo algorithm by using Kinfold [17] suggested that there is no such correlation between log MFPT and standard deviation of the number of base pairs of secondary structures in the ensemble (data not shown). This observation raises the question of which method to use for RNA folding kinetics.

In 17] the program Kinfold is described, now part of Vienna RNA Package [33, which implements the Gillespie algorithm for RNA secondary structure formation for the Turner energy model [50]. In that paper, folding time is defined to be the first passage time from the empty structure to a given target structure, so that the MFPT is expected folding time. In [4, an increase in speed of Kinfold is reported, by modifying the source code to incorporate memoization. In [14, the program KFOLD is described, which also implements 
the Gillespie algorithm, uses the Turner energy model, and performs the same elementary step base pair addition/removal transitions as does Kinfold; however, KFOLD achieves a remarkable speed-up over Kinfold by exploiting the fact that many of the base-pair addition/deletion moves and their corresponding rates do not change between each step in the simulation. As in [17, folding time is defined in [14] to be the first passage time from the empty structure to a given target structure.

Instead of determining the mean first passage time for RNA secondary structure formation by using the Monte Carlo algorithm or the Gillespie algorithm, an alternative approach is to compute the equilibrium time by solving the master equation or by simulating the Gillespie algorithm until equilibrium has been achieved. In 53 the method Treekin is described, where macrostates are defined by basins of attraction, and population occupancy curves are computed over time for each macrostate. A similar approach is described in [4], where the authors use a different method of constructing macrostates.

\section{Relation between MFPT for Markov chain versus process}

In this paper, we consider mean first passage time (MFPT) of random walks in a finite, discrete-time Markov chain $\mathbb{M}_{1}$, with transition probabilities given by equation $(2)$ in the next section. We also consider MFPT of trajectories in the finite, continuous-time Markov process $\mathbb{M}_{2}$, with transition rates given by equation (4) in the next section. Mean first passage time for the Markov chain $\mathbb{M}_{1}$ is computed by repeated simulations of the Metropolis Monte Carlo algorithm, while that for the Markov process $\mathbb{M}_{2}$ is computed by repeated simulations of the Gillespie algorithm. Although distinct, both methods to compute MFPT have been described in the literature - see summary in the previous two sections. There appears to be a tacit and sometimes explicit assumption that the kinetics results are essentially equivalent [55, 49.

Indeed, mean first passage times for protein and/or RNA folding have been computed by matrix inversion and/or Monte Carlo simulations over a Markov chain [30, 49, 7, 25, 51, 32, while the master equation and/or Gillespie simulations have been used in [17, 53, 4, 14, 56. Of particular interest is the construction of Markov state models from molecular dynamics folding trajectories for a protein or RNA molecule, followed by either mean first passage time computed by matrix inversion [51] versus equilibrium time computed by solving the master equation [56]. This leads to the question: what is the relation between mean first passage time computed by the Monte Carlo algorithm versus the Gillespie algorithm?

The answer to this question is trivial in the case that the Markov chain is $N$-regular, i.e. that each state has degree $N$ ( $N$ neighbors). In this case, we show the MFPT obtained by the time-driven Monte Carlo algorithm is equal to $N$ times that of the Gillespie algorithm. In the context of RNA secondary structures of a given sequence RNA, this suggests that MFPT determined by the time-driven Monte Carlo algorithm might be approximately equal to $\langle N\rangle$ times the MFPT determined by the Gillespie algorithm, where $\langle N\rangle$ denotes the Boltzmann expected node degree (expected number of structural neighbors), formally defined by

$$
\langle N\rangle=\sum_{s} N(s) \cdot P(s)=\sum_{s} N(s) \cdot \frac{\exp (-E(s) / R T)}{Z}
$$

where the sum is taken over all secondary structures $s$ of the RNA sequence, $N(s)$ is the number of neighbors of $s$, i.e. structures that can be obtained from $s$ by the addition or removal of a single base pair, $E(s)$ is the free energy of structure $s$ using the Turner energy model [50, $R$ the universal gas constant, $T$ absolute temperature, and $Z=\sum_{s} \exp (-E(s) / R T)$ denotes the partition function. However, we show that this is not the case, and can only conclude that the Monte Carlo and Gillespie algorithms return loosely correlated mean first passage times for RNA secondary structure folding. On the other hand, we prove that asymptotically, the expected time for a $K$-transition Monte Carlo trajectory is equal to that for a $K$-transition Gillespie trajectory multiplied by $\langle N\rangle$.

A rapidly emerging area of synthetic biology concerns the computational design of synthetic RNA [9, 13] by using inverse folding software [48, 157, 19, 15]. It seems clear that the next step in synthetic RNA design will be to control the kinetics of RNA folding. Due to the differences between MFPT computations with the Monte Carlo and Gillespie algorithms shown in this paper, we suggest that the Gillespie algorithm and related population occupancy curves determined by solution of the master equation constitute a more appropriate approach to macromolecular folding kinetics [56], rather than the Monte Carlo algorithm and related computation of mean first passage time by matrix inversion [7. In the context of synthetic RNA design, we advocate the computation of MFPT using matrix inversion for a coarse-grained model [43, as an 
efficient, initial screen of potential candidate, followed by the slower but more accurate coarse-grain method Treekin [17, 52, followed by repeated simulations using KFOLD [14].

\section{Plan of the paper}

In Section 2 we provide some background on RNA secondary structure, Markov chains and Markov processes. Section 3 describes three versions of the Monte Carlo algorithm (discrete-time time-driven, discrete-time event-driven and continuous-time event-driven) and the Gillespie algorithm, and illustates these algorithms with a simple example. Readers familiar with Markov chains, Markov processes, Monte Carlo algorithm and the Gillespie algorithm should read the pseudocode of Algorithms 1-4 (given in Figures 1-4) and otherwise skip Sections 23 and proceed directly to Section 4. That section presents a proof of a new theorem that Monte Carlo trajectory time asymptotically equals Gillespie trajectory time multiplied by the expected degree. RNA secondary structure folding simulations using the Monte Carlo algorithm and the Gillespie algorithm are given, which provide a computational illustration of the theorem. Despite the close asymptotic relation between Monte Carlo and Gillespie trajectory time, Section 4 shows that there is no such relation between Monte Carlo and Gillespie mean first passage time for RNA secondary structure folding, but only a loose correlation. Section 5 presents some discussion and concluding remarks. The Appendix presents computations that show that detailed balance does not hold for the Markov chain of secondary structures of an RNA sequence.

\section{Background}

In this section, we describe some definitions concerning RNA secondary structures, expected degree of a secondary structure network, Markov chains and Markov processes. We use the notation $\mathbb{R}$ for the set of real numbers, $\mathbb{R}^{\geq 0}$ for the set of non-negative real numbers, and $\mathbb{N}$ for the set of natureal numbers $0,1,2, \ldots$.

\subsection{Background on RNA secondary structure}

A secondary structure for an RNA nucleotide sequence $\mathbf{a}=a_{1}, \ldots, a_{n}$ is a set $s$ of Watson-Crick or wobble base pairs $(i, j)$, containing neither base triples nor pseudoknots. More formally we have the following definition.

Definition 1 A secondary structure for a given $R N A$ nucleotide sequence $a_{1}, \ldots, a_{n}$ is a set $s$ of base pairs $(i, j)$, where $1 \leq i<j \leq n$, such that:

1. if $(i, j) \in s$ then $a_{i}, a_{j}$ form either a Watson-Crick $(A U, U A, C G, G C)$ or wobble $(G U, U G)$ base pair,

2. if $(i, j) \in s$ then $j-i>\theta=3$ (a steric constraint requiring that there be at least $\theta=3$ unpaired bases between any two positions that are paired),

3. if $(i, j) \in s$ then for all $i^{\prime} \neq i$ and $j^{\prime} \neq j,\left(i^{\prime}, j\right) \notin s$ and $\left(i, j^{\prime}\right) \notin s$ (nonexistence of base triples),

4. if $(i, j) \in s$ and $(k, \ell) \in s$, then it is not the case that $i<k<j<\ell$ (nonexistence of pseudoknots).

The Turner energy model [50] is an additive energy model, where enthalpies, entropies and free energies for stacked base pairs, hairpins, bulges, internal loops, etc. are derived by least-squares fitting of experimental data from UV-absorbance (so-called optical melting) experiments, following the pioneering work of Tinoco 27. In this model, there is no energy contribution for a base pair; in contrast, stacked base pairs contribute negative, stabilizing free energy, while loop regions contribute positive, destabilizing free energy due to entropic loss. For instance, from melting experiments at $37^{\circ} \mathrm{C}$, Turner's rules assign stacking free energy of $2.08 \pm 0.06$ and enthalpy of $10.48 \pm 1.24$ to the stacked pair $\begin{array}{r}5^{\prime}-\mathrm{CU}-3^{\prime} \\ 3^{\prime}-\mathrm{GA}-5^{\prime}\end{array}$ The minimum free energy (MFE) secondary structure can be computed in time that is cubic with respect to the length of an input RNA sequence; this is done by dynamic programming following the Zuker algorithm [60, as implemented in publicly available software, such as the Vienna RNA Package [33]. 
Secondary structures can be depicted in several equivalent manners. For instance, the sequence and dot-bracket representation for a type III hammerhead ribozyme from Peach Latent Mosaic Viroid (PLMVd) AJ005312.1/282-335 is given by

GAUGAGUCUGUGCUAAGCACACUGAUGAGUCUAUGAAAUGAGACGAAACUCAUA

$.((((((.(((((\ldots)))))) \ldots \ldots((((\ldots \ldots \ldots)))) \ldots .)))))$.$) .$

The left panel of Figure 5 displays the equivalent usual presentation of the same structure, computed by RNAfold from the Vienna RNA Package [33] using the Turner 1999 energy parameters. This minimum free energy (MFE) structure agrees with the consensus structure from the Rfam database [20]. The right panel of Figure 5 depicts the network of all secondary structures for the 11-nt RNA sequence GCGCGCGCGCG, containing 27 nodes and 42 edges, where nodes are secondary structures in dot-bracket notation, and edges are indicated between each two structures $s, t$ in which $t$ is obtained by adding or removing a single base pair to/from $s$. The move set $M S_{1}$ [resp. $M S_{2}$ ] consists of adding or removing [resp. adding, removing or shifting [17, 12] ] a base pair (in this paper, we mostly concentrate on the $M S_{1}$ move set). Mean first passage time $(\mathrm{MFPT})$ for $\mathcal{M}_{1}$ [resp. $\left.\mathcal{M}_{2}\right]$ is the average first passage time from a designated initial state $x_{0}$ to a designated final state $x_{\infty}$, which can be approximated by averaging over a number of runs of the Monte Carlo algorithm [resp. Gillespie algorithm]. In the context of RNA secondary structure folding kinetics, the initial structure $x_{0}$ is often taken to be the empty structure and the target structure $x_{\infty}$ is often taken to be the MFE structure - see Figure 50 where the empty structure is indicated by a green circle, and the MFE structure by an orange circle. The contribution of this paper is to show that asymptotic trajectory times for Monte Carlo and Gillespie algorithms are related, but that mean first passage times appear to be only loosely correlated.

\subsection{Background on Markov chains and processes}

Suppose that $X=\{1, \ldots, n\}$ is a finite set of states, and for each state $x$ there is an associated energy $E(x)$. Suppose that $G$ is a graph with vertex set $X$ and an arbitrary, but fixed edge set. $G$ could be a complete graph with edges between any two distinct states, or $G$ could be the network of secondary structures for a given RNA sequence, whose vertices are the exponentially [59] many secondary structures of the sequence, and whose edges are between any two structures $x, y$ where $y$ is obtained from $x$ by removing or adding a single base pair - i.e. $y$ is obtained by a move from $M S_{1}$ from $x$, or equivalently, $x, y$ have base pair distance [36] of 1 . For state $x \in X$, let $N_{x}$ ambiguously denote either the number of immediate neighbors of $x$, or the set of immediate neighbors of $x$ in graph $G$; i.e. those $y \in X$ such that there is an edge from $x$ to $y$ belonging to $G$. Let $\mathbb{M}_{1}$ be a discrete Markov chain with set $X$ of states, having transition probability matrix $\mathbf{P}=\left(p_{x, y}\right)$ given by

$$
p_{x, y}= \begin{cases}\frac{1}{N_{x}} \cdot \min \left(1, \exp \left(-\frac{E(y)-E(x)}{R T}\right)\right) & \text { if } y \in N_{x} \\ 1-\sum_{u \in N_{x}} p_{x, u} & \text { if } x=y \\ 0 & \text { if } y \notin N_{x}, y \neq x .\end{cases}
$$

In the context of biomolecular folding (especially in Markov state models [47, 25, 39, 7, 32]), the transition matrix is used to describe protein and RNA folding kinetics. Let $\mathbf{p}(t)$ be the time-dependent population occupancy row vector, where $\mathbf{p}_{i}(t)$ is the probability that the molecule is in state $i$ at (discrete) time $t \in \mathbb{N}$ - here, state $i$ represents a particular molecular conformation in a discretized space of all possible conformations. Then

$$
\mathbf{p}(t)=\mathbf{p}(0) \cdot \mathbf{P}^{t}
$$

Let $\mathbb{M}_{2}$ be a continuous Markov process with set $X$ of states, having rate matrix $\mathbf{Q}=\left(q_{x, y}\right)$ given by

$$
q_{x, y}= \begin{cases}\min \left(1, \exp \left(-\frac{E(y)-E(x)}{R T}\right)\right) & \text { if } y \in N_{x} \\ -\sum_{u \in N_{x}} q_{x, u} & \text { if } x=y \\ 0 & \text { if } y \notin N_{x}, y \neq x\end{cases}
$$


In the context of biomolecular folding, the rate matrix is usually used in the context of the master equation, also called the Kolmogorov forward equation, defined as follows. As before, if $\mathbf{p}(t)$ denotes the time-dependent population occupancy row vector, where $\mathbf{p}_{i}(t)$ is the probability that the molecule is in state $i$ at (continuous) time $t \in \mathbb{R}$, then the master equation is the following system of ordinary differential equations

$$
\begin{aligned}
\frac{d \mathbf{p}_{1}(t)}{d t} & =\sum_{j \neq 1}\left(q_{j, 1} \mathbf{p}_{j}(t)-q_{1, j} \mathbf{p}_{1}(t)\right) \\
\frac{d \mathbf{p}_{2}(t)}{d t} & =\sum_{j \neq 2}\left(q_{j, 2} \mathbf{p}_{j}(t)-q_{2, j} \mathbf{p}_{2}(t)\right) \\
\cdots & =\sum_{j \neq n}\left(q_{j, n} \mathbf{p}_{j}(t)-q_{n, j} \mathbf{p}_{n}(t)\right)
\end{aligned}
$$

Since $q_{i, i}=-\sum_{j \neq i} q_{i, j}$, this set of equations is equivalent to the matrix differential equation

$$
\frac{d \mathbf{p}(t)}{d t}=\mathbf{p}(t) \cdot \mathbf{Q}
$$

Let $\mathcal{M}_{\mathcal{J}}$ denote the embedded Markov chain, also called jump chain, corresponding to the Markov process $\mathbb{M}_{2}$, having state space $X$ and transition probability matrix $S$ given by

$$
s_{x, y}= \begin{cases}\frac{q_{x, y}}{\sum_{z \neq x} q_{x, z}} & \text { if } y \in N_{x} \\ 0 & \text { if } x=y \text { or } y \notin N_{x} .\end{cases}
$$

The stationary probability distribution $\mathbf{p}^{*}=\left(p_{1}^{*}, \ldots, p_{n}^{*}\right)\left[\right.$ resp. $\left.\mathbf{q}^{*}=\left(q_{1}^{*}, \ldots, q_{n}^{*}\right)\right]$ for a Markov chain [resp. Markov process] with transition probability matrix $P=\left(p_{x, y}\right)$ [resp. rate matrix $Q=\left(q_{x, y}\right)$ ] must satisfy $\mathbf{p}^{*} \mathbf{P}=\mathbf{p}^{*}\left[\right.$ resp. $\left.\mathbf{q}^{*} \mathbf{Q}=\mathbf{0}\right]$. It is well-known that the stationary distribution exists and is unique for any finite, irreducible, aperiodic Markov chain [11. Detailed balance is said to hold, if for all states $x, y \in X$, $p_{x}^{*} p_{x, y}=p_{y}^{*} p_{y, x}\left[\right.$ resp. $\left.q_{x}^{*} q_{x, y}=q_{y}^{*} q_{y, x}\right]$.

Define the Boltzmann probabilities

$$
\pi_{x}=\frac{\exp (-E(x) / R T)}{Z}
$$

where the partition function $Z$ is defined by $Z=\sum_{k=1}^{n} \exp (-E(k) / R T)$. It is trivial to verify that detailed balance holds for the rate matrix $Q$ defined in equation (4). From detailed balance, it is easy to verify that the Boltzmann distribution is the stationary distribution for the Markov process defined by equation (4); indeed, if $x, y$ are neighbors and $E(y)>E(x)$, then $q_{x, y}=\exp (-(E(y)-E(x)) / R T)$ and $\pi_{x} q_{x, y}=\exp (-E(y) / R T)=$ $\pi_{y} q_{y, x}$. However, as shown in the Appendix, the Markov chain $\mathbb{M}_{1}$ does not generally satisfy detailed balance for the network of RNA secondary structures.

Using Hasting's trick [24, where in equation $\sqrt{2} p_{x, y}$ is redefined by $p_{x, y}=\frac{1}{N_{x}} \cdot \min \left(1, \exp \left(-\frac{E(y)-E(x)}{R T} \cdot \frac{N_{x}}{N_{y}}\right)\right)$ for $y \in N_{x}$, we obtain a Markov chain satisfying detailed balance. Moreover the stationary distribution for the Hastings Markov chain is easily shown to be the Boltzmann distribution from equation (7). Nevertheless, this modification comes at a computational cost, since $N_{y}$ must be determined for each neighbor $y$ of $x$. It is perhaps for this reason that Hasting's trick is not used in some kinetics simulations from the literature, as in the Monte Carlo time-driven algorithm for protein folding [40] and in Markov state models defined from long molecular dynamics (MD) trajectories [7].

\section{Monte Carlo and Gillespie algorithms}

In this section, we explore the relation between MFPT for a discrete time Markov chain with transition probability matrix (2) and that of the continuous time Markov process with rate matrix (4). Consider Algorithms 1, 2, 3 and 4. The MFPT of the Markov chain $\mathbb{M}_{1}$ can be approximated by taking the average 
over many runs of Algorithm 1 as done to show that proteins fold quickly exactly when there is a large energy gap between the energy of the native state and that of the next lowest energy misfolded state [40. Timedriven Monte Carlo Algorithm1 is equivalent to event-driven Monte Carlo Algorithm 2 since the probability of leaving state $x$ at any given time is geometrically distributed with success probability $\Phi_{1}(x)$, the probability of leaving state $x$. By replacing the geometric distribution by the continuous exponential distribution with the same mean, Monte Carlo Algorithms 2 and 3 are equivalent (see Figure 6 for a comparison of relative histograms of samples from geometric and exponential distributions having the same mean). For a given state $x$, the probability $\Phi_{1}(x)$ of leaving state $x$ is equal to the reciprocal of the number of neighbors $N_{x}$ of $x$ times the flux $\Phi_{2}(x)$ of leaving $x$. Nevertheless the jump probability $s_{x, y}$ is identical in Algorithms $2,3,4$ since $p_{x, y} / \Phi_{1}(x)$ is equal to $q_{x, y} / \Phi_{2}(x)$. It follows that Algorithms $1,2,3,4$ have identical probabilities of visiting exactly the same states in a trajectory, the only difference being that the expected waiting time in any state $x$ differs by the factor $N_{x}$ of the number of neighbors of $x$.

The previous discussion shows that the only difference between the MFPT of a discrete Markov chain and that of the related continuous time Markov process lies in the fact that the time increment $\Delta t$ in the former is sampled from a geometric distribution with mean $N_{x} / \sum_{y \in N_{x}} \min \left(1, \exp \left(-\frac{E(y)-E(x)}{R T}\right)\right)$ while the time increment $\Delta t$ in the latter is sampled from a exponential distribution with mean $1 / \sum_{y \in N_{x}}$. $\min \left(1, \exp \left(-\frac{E(y)-E(x)}{R T}\right)\right)$. Hence, if the value $N_{x}$ equals a fixed constant $N$ for each state $x$, then the Markov chain MFPT equals $N$ times the Markov process MFPT, as shown in the following example.

Example 1 Let $X=\{0,1, \ldots, 2 n-1\}$ be the set of $2 n$ states, where the energy of state $k$ is given by

$$
E(k)= \begin{cases}-3 & \text { if } k=0 \\ -2 & \text { if } k>0 \text { and } k \text { is even } \\ -1 & \text { if } k \text { is odd. }\end{cases}
$$

The left panel of Figure 7 depicts the 6 -node network $X$, where $n=3$. Let $\operatorname{Adj}(i, j)$ be the indicator function for whether $i, j$ are adjacent in a circle of size $2 n$; i.e.

$$
\operatorname{Adj}(i, j)=I[j \equiv(i \pm 1) \bmod 2 n] .
$$

Since the underlying graph is 2-regular, $N_{x}=2$ for each state $x$, and the transition probability matrix of $\mathbb{M}_{1}$ is defined by

$$
p_{i, j}= \begin{cases}0.5 \min (1, \exp (-(E(j)-E(i)))) & \text { if } \operatorname{Adj}(i, j) \\ 1-p_{i, i+1 \bmod 2 n}-p_{i, i-1} \bmod 2 n & \text { if } i=j \\ 0 & \text { otherwise }\end{cases}
$$

while the rate matrix of $\mathbb{M}_{2}$ is defined by

$$
q_{i, j}= \begin{cases}\min (1, \exp (-(E(j)-E(i)))) & \text { if } \operatorname{Adj}(i, j) \\ -p_{i, i+1 \bmod 2 n}-p_{i, i-1} \bmod 2 n & \text { if } i=j \\ 0 & \text { otherwise }\end{cases}
$$

\section{Relation between $\mathcal{M}_{1}$ and $\mathcal{M}_{2}$}

Given that the definition of the transition probability matrix $\mathbf{P}=\left(p_{x, y}\right)$ of the Markov chain $\mathcal{M}_{1}$, as given in equation (2), is similar to that of the rate matrix $\mathbf{Q}=\left(q_{x, y}\right)$ of the Markov process $\mathcal{M}_{2}$, as given in equation (4), it is natural to ask what the relation is between $\mathbf{P}$ and $\mathbf{Q}$. The answer is that there is no relation. For the Markov chain $\mathcal{M}_{1}$, equation (3) states that the population occupancy vector at time $t=1$ satisfies $\mathbf{p}(1)=\mathbf{p}(0) \cdot \mathbf{P}$. In contrast, for the Markov process $\mathcal{M}_{2}$, equation (5) states that the population occupancy vector at time $t=1$ satisfies $\mathbf{p}(1)=\mathbf{p}(0) \cdot \exp (\mathbf{Q})$, since the solution of the master equation (5) is

$$
\mathbf{p}(t)=\mathbf{p}(0) \cdot \exp (\mathbf{Q} \cdot t)
$$


For instance, if we set $n=2$ in Example 1, then the transition probability matrix is

$$
\mathbf{P}=\left(\begin{array}{llll}
0.8647 & 0.0677 & 0.0000 & 0.0677 \\
0.5000 & 0.0000 & 0.5000 & 0.0000 \\
0.0000 & 0.1839 & 0.6321 & 0.1839 \\
0.5000 & 0.0000 & 0.5000 & 0.0000
\end{array}\right)
$$

while the rate matrix is

$$
\mathbf{Q}=\left(\begin{array}{llll}
-0.2707 & 0.1353 & 0.0000 & 0.1353 \\
1.0000 & -2.0000 & 1.0000 & 0.0000 \\
0.0000 & 0.3679 & -0.7358 & 0.3679 \\
1.0000 & 0.0000 & 1.0000 & -2.0000
\end{array}\right)
$$

and the exponential of the rate matrix is

$$
\exp (\mathbf{Q})=\left(\begin{array}{llll}
0.8299 & 0.0566 & 0.0569 & 0.0566 \\
0.4183 & 0.1983 & 0.3205 & 0.0629 \\
0.1547 & 0.1179 & 0.6095 & 0.1179 \\
0.4183 & 0.0629 & 0.3205 & 0.1983
\end{array}\right)
$$

Clearly, the value $\mathbf{p}(1)=\mathbf{p}(0) \cdot \mathbf{P}$ obtained by equation (3) is unequal to the value $\mathbf{p}(1)=\mathbf{p}(0) \cdot \exp (\mathbf{Q})$ obtained by equation (5).

\section{Mean first passage time for Example 1}

Using the 20 state Markov chain obtained in Example 1 by setting $n=10$, the time to reach the minimum energy state 0 was computed for the (1) time-driven Monte Carlo Algorithm1 (2) event-driven Monte Carlo Algorithm 2 with geometrically distributed waiting times, (3) event-driven Monte Carlo Algorithm 3 with exponentially distributed waiting times, (4) Gillespie's Algorithm 4 . Times for Algorithms 1, 2 and 3 were divided by 2 , since the number $N_{x}$ of neighbors of each state $x$ is 2, while times for Algorithm 4 were reported as computed. The average time was taken over 10,000 separate runs of each algorithm, and then histograms were produced for 1000 repetitions of each of the 10,000 runs. It follows by the central limit theorem that each histogram is approximately normal; moreover, the mean and standard deviation for each histogram is reported as follows: (1) $\mu=92.94, \sigma=0.76,(2) \mu=92.97, \sigma=0.75,(3) \mu=92.96, \sigma=0.75$, (4) $\mu=92.92$, $\sigma=0.78$. The right panel of Figure 7 displays superimposed histograms of for the expected number of moves obtained on average for Algorithms 1,2,3,4 for a state set $X$ with 20 states $0,1, \ldots, 19$.

\section{Results}

\subsection{Expected trajectory time}

Throughout this section, we assume that $\mathbb{M}_{1}\left[\right.$ resp. $\left.\mathbb{M}_{2}\right]$ is a finite, irreducible, aperiodic Markov chain [resp. Markov process] whose transition probabilities $p_{x, y}$ [resp. transition rates $q_{x, y}$ ] satisfy equation (2) [resp. equation [4]]. Moreover, we assume that $\mathbb{M}_{1}$ and $\mathbb{M}_{2}$ have the same underlying set $X=\{1, \ldots, n\}$ of states, and that each state $x \in X$ is labeled by the same energy $E(x)$ in both $\mathbb{M}_{1}$ and $\mathbb{M}_{2}$.

The main result of this section is that asymptotically, for large values of $K$, the expected time taken by a $K$-transition trajectory ${ }^{2}$ for each of the Monte Carlo Algorithms 1, 2, 3 is equal to $\mu$ multiplied by the expected time for a $K$-transition trajectory of the Gillespie Algorithm 4 , where $\mu$ denotes the expected degree as defined in equation (1); i.e. the expected number of neighbors, given by

$$
\sum_{x \in X} \frac{\exp (-E(x) / R T)}{Z} \cdot N_{x}
$$

\footnotetext{
${ }^{2}$ Each step of Algorithms 2,3 and 4 involves a transition from a current state to a distinct state; however, each step of the time-driven Monte Carlo Algorithm 1 does not necessarily involve a transition to a new state, especially if the current state $x$ has low energy, so that $p_{x, x}$ may be large. For this reason, we use the term $K$-transition trajectory.
} 
Let Algorithm $1^{\dagger}, 2^{\dagger}, 3^{\dagger}, 4^{\dagger}$ respectively denote Algorithm 1,2,3,4 where line 3 of each algorithm is replaced by the line "while TRUE"; i.e. there is no condition on the while loop, so the algorithms do not terminate. In Theorem 11. we establish that for any given RNA sequence, as the number $K$ of trajectory steps approaches infinity, the trajectory time of Algorithm $3^{\dagger}$ equals that of Algorithm $4^{\dagger}$ multiplied by the expected network degree $\mu$. In the previous section, we showed that Algorithms 1,2,3 are equivalent; it follows that Algorithms $1^{\dagger}, 2^{\dagger}, 3^{\dagger}$ are also equivalent. Thus Theorem 1 directly relates (asymptotic) trajectory time of Monte Carlo time-driven and event-driven algorithms with that of Gillespie's algorithm. In order to give a formal statement of this result, we need to provide some definitions.

Definition 2 ( $K$-step trajectory and expected trajectory time) A $K$-step trajectory (sometimes called $K$-transition trajectory) of Monte Carlo Algorithm $3^{\dagger}$ [resp. Gillespie Algorithm $4^{\dagger}$ ] is a sequence $x_{0}, x_{1}, \ldots, x_{K}$ of states in $\{1, \ldots, n\}$, where $x_{i} \neq x_{i+1}$ for $i=0, \ldots, K$, although $x_{i}=x_{j}$ can occur for $|j-i| \geq 2$. Let $\tau_{3}^{K}$ [resp. $\tau_{4}^{K}$ ] denote the random variable whose value is the sum of the time increments $\Delta t$ in line 11 of Algorithm $3^{\dagger}$ [resp. Algorithm $4^{\dagger}$. Denote the expected time for $K$ steps of Algorithm $3^{\dagger}$ [resp. Algorithm $\left.4^{\dagger}\right]$ by $E\left[\tau_{3}^{K}\right]$ or $\left\langle\tau_{3}^{K}\right\rangle$ [resp. $E\left[\tau_{4}^{K}\right]$ or $\left.\left\langle\tau_{4}^{K}\right\rangle\right]$, where the expectation is taken over all stochastically sampled $K$-step trajectories (lines 13-18) and sampled time increments (line 11) of Algorithm $3^{\dagger}$ [resp. Algorithm $\left.4^{\dagger}\right]$.

Theorem 1 Let $\mu=\sum_{x \in X} \frac{\exp (-E(x) / R T)}{Z} \cdot N_{x}$ denote the Boltzmann expected network degree, where $N_{x}$ denotes the degree of state $x$. Then $\lim _{K \rightarrow \infty} \frac{E\left[\tau_{1}^{K}\right]}{E\left[\tau_{2}^{K}\right]}=\mu$.

Proof: For each state $x \in X$, define the Boltzmann probability $\pi_{x}$ is defined by $\pi_{x}=\frac{\exp (-E(x) / R T)}{Z}$, where the partition function $Z$ is defined by $\sum_{x \in X} \exp (-E(x) / R T)$. For state $x \in X$, define the probability of leaving $x$ [resp. flux out of $x]$, denoted $\Phi_{1}(x)\left[\right.$ resp. $\left.\Phi_{2}(x)\right]$ by

$$
\begin{array}{r}
\Phi_{1}(x)=\sum_{y \neq x} p_{x, y}=\frac{\Phi_{2}(x)}{N_{x}} \\
\Phi_{2}(x)=\sum_{y \neq x} q_{x, y}=N_{x} \cdot \Phi_{1}(x)
\end{array}
$$

Recall that the transition probability matrix $S$ of the embedded chain, also called jump chain, $\mathbb{M}_{J}$ is defined in equation (6), and hence satisfies

$$
s_{x, y}= \begin{cases}\frac{q_{x, y}}{\Phi_{2}(x)}=\frac{p_{x, y}}{\Phi_{1}(x)} & \text { if } y \in N_{x} \\ 0 & \text { if } x=y \text { or } y \notin N_{x}\end{cases}
$$

A $K$-step trajectory of either Algorithm $3^{\dagger}$ or $4^{\dagger}$ corresponds to a random walk on the jump chain with states $1, \ldots, n$ and transition matrix $S$ as defined in equation (15). Since the rate matrix $Q$, defined in equation (4), for the Markov process $\mathbb{M}_{2}$ corresponding to Algorithm $4^{\dagger}$ trivially satisfies detailed balance, so does the transition matrix $S$ for the jump chain, defined in equation 15 .

Claim: Define the row vector $s^{*}=\left(s_{1}^{*}, \ldots, s_{n}^{*}\right)$ by

$$
s_{x}^{*}=\frac{q_{x}^{*} \Phi_{2}(x)}{\sum_{y \in \mathbb{S S}(\mathbf{a})} q_{y}^{*} \Phi_{2}(y)} .
$$

Then $s^{*} S=s^{*}$, hence $s^{*}$ is the (unique) stationary distribution for the jump chain $\mathbb{M}_{J}$. 
Proof: For fixed state $y \in\{1, \ldots, n\}$, the $y$ th coordinate of the row vector $s^{*} S$ satisfies

$$
\begin{aligned}
\left(s^{*} S\right)_{y} & =\sum_{x=1}^{n} s_{x}^{*} \cdot s_{x, y}=\sum_{x \neq y} s_{x}^{*} \cdot s_{x, y} \\
& =\sum_{x \neq y}\left(\frac{q_{x}^{*} \Phi_{2}(x)}{\sum_{z \in \mathbb{S S}(\mathbf{a})} q_{z}^{*} \Phi_{2}(z)}\right) \cdot \frac{q_{x, y}}{\Phi_{2}(x)} \\
& =\sum_{x \neq y} \frac{q_{x}^{*} q_{x, y}}{\sum_{z \in \mathbb{S S}(\mathbf{a})} q_{z}^{*} \Phi_{2}(z)}=\sum_{x \neq y} \frac{q_{y}^{*} q_{y, x}}{\sum_{z \in \mathbb{S S}(\mathbf{a})} q_{z}^{*} \Phi_{2}(z)} \\
& =q_{y}^{*} \cdot\left(\sum_{x \neq y} q_{y, x}\right) \frac{1}{\sum_{z \in \mathbb{S S}(\mathbf{a})} q_{z}^{*} \Phi_{2}(z)} \\
& =\frac{q_{y}^{*} \Phi_{2}(y)}{\sum_{z \in \mathbb{S S}(\mathbf{a})} q_{z}^{*} \Phi_{2}(z)}=s_{y}^{*}
\end{aligned}
$$

Note that line 2 follows by definition of $s_{x}^{*}$ and $s_{x, y}$; line 3 follows by detailed balance of $q_{x}^{*} q_{x, y}=q_{y}^{*} q_{y, x}$; line 4 follows by factoring out terms that do not depend on $x$, and line 5 follows by the definition of $\Phi_{2}(y)$.

An equivalent, more intuitive statement of equation $\sqrt{16}$ is that $s_{y}^{*}$ equals the the Boltzmann probability $q_{y}^{*}$ of state $y$, times the flux $\Phi_{2}(y)$ out of $y$, divided by the expected flux with respect to the Boltzmann distribution; i.e. $s_{y}^{*}$ is the ratio of the Boltzmann weighted flux out of $y$ with respect to the expected flux out of any state, where expectation is taken over all states. This proves the claim.

If the number $K$ of steps in the trajectory is large, then the expected number of occurrences of each state $x$ is $s_{x}^{*} K$. The expected time to leave state $x$ in Algorithm $3^{\dagger}$ [resp. Algorithm $\left.4^{\dagger}\right]$ is $\frac{1}{\Phi_{1}(x)}\left[\right.$ resp. $\left.\frac{1}{\Phi_{2}(x)}\right]$, since waiting times are exponentially distributed. It follows that the ratio of the expected time for a $K$-step trajectory of Algorithm $3^{\dagger}$ divided by the expected time for a $K$-step trajectory of Algorithm $4^{\dagger}$ equals:

$$
\begin{aligned}
\frac{E\left[\tau_{1}^{K}\right]}{E\left[\tau_{2}^{K}\right]} & =\frac{\sum_{x=1}^{n} \frac{s_{x}^{*} K}{\Phi_{1}(x)}}{\sum_{x=1}^{n} \frac{s_{x}^{*} K}{\Phi_{2}(x)}}=\frac{\sum_{x=1}^{n} \frac{s_{x}^{*} N_{x}}{\Phi_{2}(x)}}{\sum_{x=1}^{n} \frac{s_{x}^{*}}{\Phi_{2}(x)}} \\
& =\frac{\sum_{x=1}^{n} \frac{q_{x}^{*} \Phi_{2}(x)}{\sum_{z} q_{z}^{*} \Phi_{2}(z)} \cdot \frac{N_{x}}{\Phi_{2}(x)}}{\sum_{x=1}^{n} \frac{q_{x}^{*} \Phi_{2}(x)}{\sum_{z} q_{z}^{*} \Phi_{2}(z)} \cdot \frac{1}{\Phi_{2}(x)}} \\
& =\frac{\sum_{x=1}^{n} q_{x}^{*} N_{x}}{\sum_{x=1}^{n} q_{x}^{*}}=\frac{\sum_{x=1}^{n} \pi_{x} N_{x}}{1} \\
& =\sum_{x=1}^{n} \frac{\exp (-E(x) / R T) \cdot N_{x}}{Z}=\mu(\mathbf{a}) .
\end{aligned}
$$

This completes the proof of Theorem 1

Corollary 1 The same proof shows that mean recurrence time for Markov chain $\mathbb{M}_{1}$ is equal to $\mu$ times the mean recurrence time for Markov process $\mathbb{M}_{2}$. (See [16] for the definition of mean recurrence time.)

\subsection{Illustrative examples from RNA}

For a fixed RNA sequence $\mathbf{a}=a_{1}, \ldots, a_{m}$, denote the set of all secondary structures for a by $\mathbb{S S}(\mathbf{a})$. Define the Markov chain $\mathbb{M}_{1}$ [resp. Markov process $\left.\mathbb{M}_{2}\right]$ for the set $\mathbb{S S}(\mathbf{a})$ of states, where transitions $s \rightarrow t$ occur

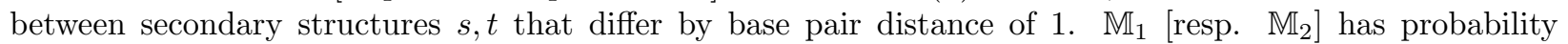
matrix $P=\left(p_{x, y}\right)$ [resp. rate matrix $Q=\left(q_{x, y}\right)$ ] defined by equation (2) [resp. equation (4)], where $E(x)$ denotes the free energy of secondary structure $x$ with respect to the Turner energy model [50]. Theorem 1 then implies that for trajectories of length $K$, for large $K$, the expected trajectory time $E\left[\tau_{1}^{K}\right]$ for each of Algorithm $1^{\dagger}$, Algorithm $2^{\dagger}$, and Algorithm $3^{\dagger}$ is approximately $\mu$ multiplied by the trajectory time $E\left[\tau_{2}^{K}\right]$ 
for Algorithm $4^{\dagger}$, where $\mu$ is the expected degree for the network $\mathbb{S S}(\mathbf{a})$ of secondary structures for RNA sequence $\mathbf{a}=a_{1}, \ldots, a_{n}$.

Although the set $\mathbb{S S}(\mathbf{a})$ is generally of size exponential in $n$ (see [46, 18), we recently described a cubic time dynamic programming algorithm [10] to compute the expected network degree of $\mathbb{S}(\mathbf{a})$

$$
\mu(\mathbf{a})=\sum_{x \in \mathbb{S S}(\mathbf{a})} \frac{\exp (-E(x) / R T)}{Z} \cdot N_{x}
$$

where $N_{x}$ is the set of secondary structures $y$ having base pair distance 1 to $x$.

We illustrate Theorem 1 in the following computational experiments. For a given RNA sequence a, let $x_{0}$ denote the empty secondary structure containing no base pairs. If algorithms $\mathrm{C}^{\dagger}$ and $\mathrm{D}^{\dagger}$ begin from the same initial state $x_{0}$ and use the same pseudo-random number generator seed, then the algorithms are said to be synchronized, or to generate synchronized trajectories; if no random seed is set, then the algorithms are said to be unsynchronized. If Algorithms $3^{\dagger}$ and $4^{\dagger}$ are synchronized, then clearly each algorithm visits exactly the same states in the same order.

The following computational experiment is described. Given an RNA sequence, $\mathbf{a}=a_{1}, \ldots, a_{n}$, we determine the minimum free energy (MFE) structure $s^{*}$ by Zuker's algorithm [60] as implemented in Vienna RNA Package 33. For the $32 \mathrm{nt}$ fruA SECIS element CCUCGAGGGG AACCCGAAAG GGACCCGAGA GG, for which the target minimum free energy structure is reached generally in less than one thousand moves, we analyzed a trajectory of one million moves. For synchronized runs of Algorithm $3^{\dagger}\left[\right.$ resp. Algorithm $\left.4^{\dagger}\right]$ the trajectory time was approximately $98.16168 \cdot 10^{9}$ [resp. 9.82994 $\cdot 10^{9}$ ] expected number of neighbors $\mu(\mathbf{a})=10.00146$, and the ratio of Algorithm $3^{\dagger}$ trajectory time divided by $\mu(\mathbf{a})$ is $9.81474 \cdot 10^{9}$ - close to the Algorithm $4^{\dagger}$ trajectory time. For nonsynchronized runs of Algorithm $3^{\dagger}$ [resp. Algorithm $4^{\dagger}$ ] the trajectory time was approximately $98.03194 \cdot 10^{9}$ [reps. 9.78867 $\cdot 10^{9}$ ], and the ratio of Algorthm $\mathrm{C}^{\dagger}$ trajectory time divided by $\mu(\mathbf{a})$ is $9.80177 \cdot 10^{9}$ - very close to Algorithm $4^{\dagger}$ trajectory time. Similar validation of Theorem 1 was shown in second computational experiment, where a 500 thousand step trajectory was generated for the 76 nt ala-tRNA from Mycoplasma mycoides with Sprinzl ID RA1180 (tRNAdb ID tdbR00000006) [29] using Algorithms $\mathrm{C}^{\dagger}$ and $\mathrm{D}^{\dagger}$. A final illustration of Theorem 1 is given in the right panel of Figure 7, which displays a scatter plot, for both synchronized and unsynchronized runs of one million steps for the Monte Carlo Algorithm $3^{\dagger}$ and the Gillespie Algorithm $4^{\dagger}$ for forty $20 \mathrm{nt}$ randomly generated RNA sequences, each of whose MFE secondary structure has free energy $E<-2.5 \mathrm{kcal} / \mathrm{mol}$, each having at most 2500 secondary structures, and each with expected compositional frequency of 0.25 for each of A,C,G,U.

Figure 8 shows the distribution for the number of neighbors the 76 nt transfer RNA (RA1180 from tRNAdb 2009 [29]), with mean 29.29 and standard deviation 4.14, as well as that for the $56 \mathrm{nt}$ spliced leader RNA from Leptomonas collosoma (a known conformational switch), with mean 70.05 and standard deviation of 33.84. Although we can exactly compute the expected network degree in seconds [10, the only current method to obtain an approximation of the distribution is by sampling using RNAsubopt [54] from the Vienna RNA Package [33, a computation requiring many hours. It follows that the RNA examples illustrating Theorem 1 could not have been given without use of the dynamic programming algorithm to compute expected network degree [10].

Remark 1 In our examples of RNA secondary structure trajectory time, we considered elementary step transitions between secondary structures $x, y$, in which $y$ is obtained from $x$ by addition or removal of $a$ single base pair. However, Theorem 1 is equally applicable for more general move sets between RNA secondary structures, including shift moves [17] and even the addition and removal of entire stems [28]. In each of these cases, it is easily established that the underlying Markov chain [resp. Markov process] is finite, irreducible and aperiodic, so that Theorem 1 applies.

Remark 2 The program Kinfold [17] is an implementation of the Gillespie algorithm, which supports both move set $M S_{1}$, consisting of adding or removing a single base pair, and move set $M S_{2}$, consisting of adding, removing, or shifting a base pair. Since we found it difficult to modify the source code of Kinfold to implement the Monte Carlo algorithm, we implemented the Monte Carlo and Gillespie algorithms for RNA secondary structure networks in a C-program mc.c, available at http: //bioinformatics. bc. edu/ clotelab/RNAexpNumNbors/. In [12], we generalized the algorithm from [10] to efficiently compute the 
expected network degree for $R N A$ secondary structure networks with respect to move set $M S_{2}$. At the present time, however, our program mc.c does not support shift moves.

\subsection{Monte Carlo and Gillespie MFPT}

Here, we show that unless the network is regular (each node has the same degree), there is no simple analogue of Theorem 1 to relate the mean first passage time (MFPT) for the Monte Carlo Algorithm 3 and the Gillespie Algorithm 4 for RNA secondary structure folding kinetics. Algorithms $3^{\dagger}$ and $4^{\dagger}$ differ from Algorithms 3 and 4 , in that the former algorithms do not terminate computation upon visiting the minimum free energy structure $x_{\infty}$. This modification allowed us to establish Theorem 1 .

In Example 1 from Section 3 , we described the computation of mean first passage time (MFPT) using each of Algorithms 1,2,3,4 for a Markov chain $\mathbb{M}_{1}$ [resp. Markov process $\mathbb{M}_{2}$ ], having states $2 n$ states $0,1, \ldots, 2 n-1$, where energy $E(x)$ of state $x$ is defined in equation (8), and transition probability $p_{x, y}$ of moving from state $x$ to state $y \in\{x-1 \bmod 2 n, x+1 \bmod 2 n\}$ is defined in equation (10). Since the underlying graph is 2-regular, Figure 7 indeed shows that MFPT for each of Monte Carlo Algorithms 1,2,3 is twice the MFPT of Gillespie Algorithm 4.

By slightly modifying the topology of Example 1 , we obtain a non-regular network with states $0, \ldots, 2 n-1$, for which all states have degree 2, with the exception of the (terminal) minimum energy state 0 and the last state $2 n-1$. For this example, described in the following, there appears to be no simple relation between the MFPT of Monte Carlo Algorithms 1,2,3 with that of Gillespie Algorithm 4.

Example 2 Define Markov chain $\mathbb{M}_{1}$ [resp. Markov process $\mathbb{M}_{2}$ ] as in Example[1, with the sole exception that state 0 is no longer connected to state $2 n-1$; i.e. the indicator function Adj $(i, j)$ for whether $i, j$ are adjacent is redefined by

$$
\operatorname{Adj}(i, j)= \begin{cases}1 & \text { if } 0 \leq i<2 n-1 \text { and } j=i+1 \\ 1 & \text { if } 0 \leq j<2 n-1 \text { and } i=j+1 \\ 0 & \text { otherwise }\end{cases}
$$

Transition probabilities [resp. rates] are defined by equation (2) [resp. equation (4)] where we set RT $=1$, hence

$$
p_{i, j}= \begin{cases}0.5 \cdot \min (1, \exp (-(E(j)-E(i)))) & \text { if } \operatorname{Adj}(i, j), 1 \leq i, j<2 n-1 \\ \min (1, \exp (-(E(j)-E(i)))) & \text { if } i=0, j=1 \text { or } i=2 n-1, j=2 n-2 \\ 1-p_{i,(i+1 \bmod 2 n)}-p_{i,(i-1 \bmod 2 n)} & \text { if } i=j \\ 0 & \text { otherwise }\end{cases}
$$

while the rate matrix of $\mathbb{M}_{2}$ remains unmodified, defined by

$$
q_{i, j}= \begin{cases}\min (1, \exp (-(E(j)-E(i)))) & \text { if } \operatorname{Adj}(i, j) \\ -p_{i,(i+1 \bmod 2 n)}-p_{i,(i-1 \bmod 2 n)} & \text { if } i=j \\ 0 & \text { otherwise }\end{cases}
$$

Let $n=10$, so that there are 20 states $0, \ldots, 19$, and let the initial state $x_{0}=10$, as in Example 1 . The time to reach the minimum energy state 0 was computed for the time-driven Monte Carlo Algorithm 1, eventdriven Monte Carlo Algorithm 2 with geometrically distributed waiting times, event-driven Monte Carlo Algorithm 3 with exponentially distributed waiting times and the Gillespie Algorithm 4. The average time was taken over 10,000 separate runs of each algorithm, and histograms were produced for 1000 repetitions of each of the 10,000 runs. The mean and standard deviation for each histogram is reported as follows: (1) $\mu=520.93, \sigma=16.99$, (2) $\mu=520.52, \sigma=16.97$, (3) $\mu=520.67, \sigma=17.00$, (4) $\mu=265.21, \sigma=8.62$. Figure 9 displays the relative histograms for this data, where for comparison purposes, first passage times for Gillespie Algorithm 4 are multiplied by 2. The uniform expected number of neighbors, or network degree, computed over the the collection of 19 non-terminal states $x \neq 0$, is 1.947 , while the Boltzmann expected number of neighbors is 1.710 . In contrast, the ratio of the average 520.706 of the mean first passage times computed by Monte Carlo Algorithms 1,2,3 divided by the mean first passage time 265.209 for the Gillespie 
Algorithm 4 is equal to 1.963. The p-value for the 2-tailed T-test for equality of Monte Carlo MFTP 520.706 with Gillespie MFPT 265.21 times uniform network degree 1.947 is $1.93149 \cdot 10^{-11}$. It follows that Monte Carlo MFPT is statistically different from Gillespie MFPT times the expected degree of the network in the case of Example 2 (for either uniform or Boltzmann probability).

\subsection{Folding trajectories for the 10-mer GGGGGCCCCC}

Theorem 1 implies that IF there is no termination condition for Algorithms 3 and 4 (obtained by replacing line 3 by while TRUE), so that every secondary structure of the 10-mer GGGGGCCCCC may be visited multiple times (including the MFE structure), THEN for a sufficiently large number of algorithm steps $K$, the total time for the trajectory of Algorithm 3 is (asymptotically) equal to the total time for the trajectory of Algorithm 4 times the expected network degree. We now show that this is false for mean first passage times; i.e. if Algorithms 3 and 4 terminate upon reaching the MFE structure, then there is no such relation between their trajectory times, which correspond to the first passage time from the empty initial structure to the MFE structure. Before proceeding, we need some notation.

Definition 3 (Expected convergence time) Given an $R N A$ sequence $\mathbf{a}=a_{1}, \ldots, a_{n}$, let $\tau_{3}^{M F E}(\mathbf{a})$ [resp . $\left.\tau_{4}^{M F E}(\mathbf{a})\right]$ denote the random variable whose value is the sum of the time increments $\Delta t$ in line 11 of Algorithm 3 [resp. Algorithm 4] until the algorithm converges, where initial state $x_{0}$ is the empty secondary structure of $\mathbf{a}$ and final state $x_{\infty}$ is the MFE structure of $\mathbf{a}$. Let $E\left[\tau_{3}^{M F E}(\mathbf{a})\right]$ or $\left\langle\tau_{3}^{M F E}(\mathbf{a})\right\rangle$ [resp. $E\left[\tau_{4}^{M F E}(\mathbf{a})\right]$ or $\left.\left\langle\tau_{4}^{M F E}(\mathbf{a})\right\rangle\right]$ denote the expected convergence time of Algorithm 3 [resp. Algorithn 4], or in other words, the mean first passage time to fold the RNA sequence a using Monte Carlo Algorithm 3 [resp. Gillespie Algorithm 4].

For the 10 nt RNA sequence GGGGGCCCCC, we ran Algorithms 3 and 4 to compute the trajectory time for 100,000 distinct, synchronized folding trajectories, each trajectory starting from the empty initial structure $x_{0}=\emptyset$ and terminating upon reaching the minimum free energy (MFE) structure $x_{\infty}=$

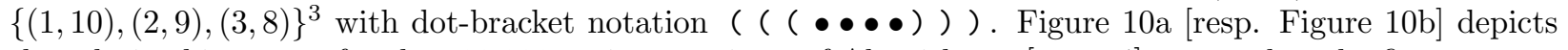
the relative histogram for the 100,000 trajectory times of Algorithm 3 [resp. 4] - note that the first passage times appear to be exponentially distributed, although the fit is not good. Since the folding experiments were synchronized, for each $i=1, \ldots, 100,000$, the number of steps taken by each of Algorithms 3 and 4 were identical, as were the secondary structures visited in each step, so that the only difference between Algorithms 3 and 4 consisted in the incremental times $\Delta t$ determined in line 11 of each algorithm, as well as in the total trajectory time in line 20 of each algorithm.

\section{Expected degree with respect to the Boltzmann distribution}

Using the software from [10, we find that Boltzmann expected number of neighbors $\langle N\rangle$, as defined in equation (1), for the set of all 62 secondary structures of the 10-mer GGGGGCCCCC is $\langle N\rangle=3.031162 \approx$ 3.03. Figure $10 k$ depicts the relative histogram for the pairwise differences $T_{c}-\langle N\rangle \cdot T_{d}$. Let the null hypothesis $H_{0}$ assert that trajectory time $T_{c}$ for Algorithm 3 to reach the MFE structure is equal to $\langle N\rangle$ multiplied by the trajectory time $T_{d}$ for Algorithm 4 to reach the MFE structure; i.e. $H_{0}$ is the assertion that $T_{c}=3.031162 \cdot T_{d}$.

The p-value for $n=100,000$ paired values $\left(T_{c},\langle N\rangle \cdot T_{d}\right)$ is $9.0923 E-107$, since the mean $\bar{x}_{d i f}$ of paired differences $T_{c}-\langle N\rangle \cdot T_{d}$ satisfies $\bar{x}_{d i f}=1277.20=\sum_{i=1}^{n} \frac{T_{c}[i]-\langle N\rangle \cdot T_{d}[i]}{n}$, the standard deviation $s_{d i f}$ of the paired differences satisfies $s_{d i f}=18379.89$, the test statistic $t=21.97=\frac{\bar{x}_{d i f}}{s_{d i f} / \sqrt{n}}$, and the number $d f$ of degrees of freedom $n-1=99,999$.

For $\alpha=0.05$, to compute the $1-\alpha=95 \%$ confidence interval for $T_{c}-\langle N\rangle \cdot T_{d}$, we determine the margin of error $E=t_{\alpha / 2} \cdot \frac{s_{d i f}}{\sqrt{n}} \approx 1.645 \cdot 58.12=95.60$, yielding a confidence interval of $\left(\bar{x}_{d i f}-E, \bar{x}_{d i f}+E\right)=$ $(1181.59,1372.80)$. Under the null hypothesis, $\bar{x}_{d i f}$ is asserted to be 0 , which does not belong to the confidence interval. Even repeating the computation with $\alpha=10^{-100}$, we still observe that 0 does not belong to the corresponding confidence interval $(39.33,2515.06)$. Since Algorithm 3 first passage times are greater than expected network degree time Algorithm 4 first passage times, it must be that a disproportionately large set

\footnotetext{
${ }^{3}$ Recall that secondary structures may be represented as a set of base pairs.
} 
of the secondary structures visited in folding trajectories of the 10-mer GGGGGCCCCC have larger degree (a larger number of neighboring structures that can be reached by the addition or removal of a single base pair) than the network average. This suggests that the (identical) folding trajectories of Algorithms 3 and 4 do not visit mainly low energy structures, although our detailed analysis of one 10 nt RNA sequence can hardly be representative.

Note as well that the paired times $T_{c}$ and $\langle N\rangle \cdot T_{d}$ are poorly correlated. Since first passage times are not normally distributed, we compute the Spearman correlation of 0.439397 ; although Pearson correlation should not be used for non-normal distributions, the Pearson correlation is 0.445950 . Analysis of the same data after removal of outliers (e.g. computing the $10 \%$-trimmed mean, etc.) even strengthen the conclusions just reached (data not shown). It follows that the analogue of Theorem 1 for trajectories that terminate when reaching the MFE does not hold - moreover, the correlation is low for the paired trajectory times for synchronized Algorithms 3 and 4 to reach the MFE structure. Since tremendous computational resources would be required to perform a similar analysis for longer RNA sequences, we restrict our attention to the correlation between the synchronized and non-synchronized first passage times for

Algorithms 3 (continuous-time event-driven Monte Carlo) and 4 (Gillespie) - see Tables 1 and 2 .

\section{Expected degree with respect to the uniform distribution}

Our software [10] also computes that the uniform expected number of neighbors $\langle N\rangle=3.548387 \approx 3.55$ for the set of all 62 secondary structures of the 10-mer GGGGGCCCCC, defined by equation (1) where the free energy $E(s)$ of every secondary structure is defined to be zero, or equivalently by setting probability $P(s)=\frac{1}{Z}=\frac{1}{62}$, where $Z$ now denotes the the total number of secondary structures of GGGGGCCCCC. We now re-analyze the 100,000 first passage times of Algorithms 3 and 4 .

Recall that the null hypothesis $H_{0}$ asserts that trajectory time $T_{c}$ for Algorithm 3 to reach the MFE structure is equal to $\langle N\rangle$ multiplied by the trajectory time $T_{d}$ for Algorithm 4 to reach the MFE structure, except that we now use uniform expected number of neighbors; i.e. $H_{0}$ is the assertion that $T_{c}=3.548387 \cdot T_{d}$.

The p-value for $n=100,000$ paired values $\left(T_{c},\langle N\rangle \cdot T_{d}\right)$ is $3.42447 E-09$. For $\alpha=0.05$, to compute the $1-\alpha=95 \%$ confidence interval for $T_{c}-\langle N\rangle \cdot T_{d}$, we determine the $95 \%$ confidence interval of $\left(\bar{x}_{d i f}-\right.$ $\left.E, \bar{x}_{d i f}+E\right)=(-478.01,-269.87)$. The $95 \%$ confidence interval for an independent run of Algorithms 3 and 4 to generate another set of 100,000 first passage times was $(-389.30,-362.03)$. It follows that the uniform probability analogue of Theorem 1 for trajectories that terminate when reaching the MFE does not hold.

Since Algorithm 3 first passage times are now less than the uniform expected number of neighbors multiplied by Algorithm 4 first passage times, it must be that a a disproportionately large set of the secondary structures which are visited in folding trajectories of the 10-mer GGGGGCCCCC have lower degree (a lower number of neighboring structures that can be reached by the addition or removal of a single base pair) than the network average. This suggests that the (identical) folding trajectories of Algorithms 3 and 4 do not visit mainly high energy structures (having few base pairs, hence potentially a larger number of neighbors), although our detailed analysis of one $10 \mathrm{nt}$ RNA sequence can hardly be representative.

Finally, we note that the ratio of the mean first passage time $\left\langle T_{c}\right\rangle$ [resp. $\left.\left\langle T_{d}\right\rangle\right]$, taken over $n=100,000$ runs of Algorithm 3 [resp. Algorithm 4] is $\frac{\left\langle T_{c}\right\rangle}{\left\langle T_{d}\right\rangle} \approx 3.43$, a value that appears somewhat closer to uniform expected network degree of 3.548387 than the Boltzmann expected network degree of 3.031162.

\section{Correlation analysis for 20-mers}

In the case of RNA secondary structure folding kinetics, it appears that Gillespie MFPT times expected network degree is even less correlated with Monte Carlo MFPT than unaltered Gillespie MFPT. Table 1 shows the Pearson correlation between the averages, taken over 1000 runs, of the MFPT for each of 100020 nt RNAs, when computed by the Monte Carlo (MC) method (Algorithm $3^{\dagger}$ ), synchronized (syn) [resp. not synchronized (ns)] with Gillespie's (G) method (Algorithm $4^{\dagger}$ ). Additionally, we performed two repetitions of the unsyncronized runs, designated experiment A and B in the table. For these data, the MFPT computed by Monte Carlo is highly correlated with that computed by Gillespie: G (syn) has correlation of 0.85702 with MC (syn) and a correlation of 0.81030 with MC (ns). Surprisingly, G (syn) has a somewhat lower orrelation of 0.85381 , for $\mathrm{MC}^{*}$ (syn), obtained by dividing the $\mathrm{MC}$ (syn) time for each sequence by its expected number of neighbors; similarly, G (syn) has a somewhat lower correlation of 0.80559 for $\mathrm{MC}^{*}(\mathrm{~ns})$, 


\begin{tabular}{|l|llllll|}
\hline & MC (syn) & MC (ns A) & MC (ns B) & G (syn) & G (ns A) & G (ns B) \\
\hline MC (syn) & 1.000000 & 0.994405 & 0.981810 & 0.857019 & 0.871860 & 0.880024 \\
MC (ns A) & 0.994405 & 1.000000 & 0.989948 & 0.810297 & 0.830360 & 0.839469 \\
MC (ns B) & 0.981810 & 0.989948 & 1.000000 & 0.783331 & 0.809399 & 0.814758 \\
G (syn) & 0.857019 & 0.810297 & 0.783331 & 1.000000 & 0.996953 & 0.996666 \\
G (ns A) & 0.871860 & 0.830360 & 0.809399 & 0.996953 & 1.000000 & 0.998148 \\
G (ns B) & 0.880024 & 0.839469 & 0.814758 & 0.996666 & 0.998148 & 1.000000 \\
\hline
\end{tabular}

Table 1: Correlation shown by folding time averages, taken over 1000 runs, for $100020 \mathrm{nt}$ random sequences taken from the benchmarking set of 43. Abbreviations are as follows. MC (sym): synchronized Monte Carlo Algorithm $3^{\dagger}$; MC (ns A): batch A for nonsynchronized Monte Carlo Algorithm $3^{\dagger}$; MC (ns B): batch B for nonsynchronized Monte Carlo Algorithm $3^{\dagger}$; G (sym): synchronized Gillespie Algorithm $4^{\dagger}$; G (ns A): batch A for nonsynchronized Gillespie Algorithm $4^{\dagger}$; G (ns B): batch B for nonsynchronized Gillespie Algorithm $4^{\dagger}$.

\begin{tabular}{|l|llllll|}
\hline & $\mathrm{MC}($ syn) & $\mathrm{MC}(\mathrm{ns})$ & $\mathrm{G}(\mathrm{syn})$ & $\mathrm{G}(\mathrm{ns})$ & $\mathrm{MC}(\mathrm{syn})$ & $\mathrm{MC}(\mathrm{ns})$ \\
\hline $\mathrm{MC}(\mathrm{syn})$ & 1.000000 & 0.994405 & 0.857019 & 0.871860 & 0.985542 & 0.978662 \\
$\mathrm{MC}(\mathrm{ns})$ & 0.994405 & 1.000000 & 0.810297 & 0.830360 & 0.981410 & 0.985471 \\
$\mathrm{G}(\mathrm{syn})$ & 0.857019 & 0.810297 & 1.000000 & 0.996953 & 0.853810 & 0.805594 \\
$\mathrm{G}(\mathrm{ns})$ & 0.871860 & 0.830360 & 0.996953 & 1.000000 & 0.871133 & 0.827705 \\
MC*(syn) $^{*}$ & 0.985542 & 0.981410 & 0.853810 & 0.871133 & 1.000000 & 0.994563 \\
$\mathrm{MC}^{*}(\mathrm{~ns})$ & 0.978662 & 0.985471 & 0.805594 & 0.827705 & 0.994563 & 1.000000 \\
\hline
\end{tabular}

Table 2: Correlation shown by folding time averages, taken over 1000 runs, for $100020 \mathrm{nt}$ random sequences taken from the benchmarking set of 43 . Abbreviations are as in Table 1 , with the addition that $\mathrm{MC}^{*}$ (syn) [resp. MC* (ns)] is produced by dividing the Monte Carlo (Algorithm $3^{\dagger}$ ) folding time by the expected sequence connectivity for synchronized [resp. nonsynchronized] computations.

obtained by dividing the MC (ns) time for each sequence by its expected number of neighbors. In other words, despite the fact that the ratio of Monte Carlo trajectory time over Gillespie trajectory time equals the expected number of neighbors for sufficiently long trajectories as proved in Theorem 1 1 , there is no such relation between Monte Carlo MFPT and Gillespie MFPT, presumably since the mean first passage time is generally reached within $K$ steps, where $K$ is too small for the asymptotic effects of Theorem 1 to apply.

\section{Discussion}

In this paper, we have compared Markov chains and related Markov processes by considering four closely related Algorithms 1,2,3,4. If the underlying graph for the Markov chain $\mathbb{M}$ is $N$-regular, so that each state of $\mathbb{M}$ has exactly $N$ neighbors, then it follows that on average, mean first passage time (MFPT) computed by each of the Monte Carlo Algorithms 1,2 and 3 equals $N$ multiplied by the MFTP computed by Gillespie Algorithm 4. Although the Markov chain of RNA secondary structures of a given RNA sequence is generally not $N$-regular for any $N$, the total time along a Monte Carlo trajectory is asymptotically equal to the Boltzmann expected number of neighbors $\langle N\rangle=\sum_{s} \frac{\exp (-E(s) / R T)}{Z} \cdot N(s)$ multiplied by the total time along a Gillespie trajectory, as proved in Theorem 1. Computational experiments on several RNAs confirm this result, provided that trajectories are sufficiently long to exhibit asymptotic properties of Markov chains. Our code mc.c for Algorithms 3 and 4, is written in $\mathrm{C}$ and makes calls to the function energy_of_structure() from libRNA . a of Vienna RNA Package [33. This program, along with C programs to compute the expected network degree for RNA secondary structures with move set $M S_{1}$ (base pair addition or deletion) [10] or with move set $M S_{2}$ (base pair addition, deletion or shift) [12, is publicly available at http://bioinformatics.bc.edu/clotelab/RNAexpNumNbors.

Since Anfinsen's pioneering experimental result on the folding of bovine ribonuclease [3, it is widely accepted that the native state of a biomolecule is a free energy minimum. In the literature on biomolecular folding, there is sometimes a tacit assumption that kinetics simulations using the Monte Carlo and Gillespie 
algorithms yield comparable results. Indeed, in [55] Tang et al. assert that "We demonstrate with two different RNA that the different analysis methods (ME, MC, MMC) produce comparable results and can be used interchangeably 4 , The results of this paper suggest that one should not make tacit assumptions concerning folding kinetics simulations using Monte Carlo and Gillespie algorithms, when different nodes in the network have different numbers of neighbors, as in the case for RNA secondary structures.

If computing the mean first passage time requires sufficiently long trajectories, then we would expect Monte Carlo MFPT to approximately equal Gillespie MFPT times the expected number of neighbors. However, for a slight modification of Example 1 described in Example 2, as well as for a benchmarking set of 100020 nt RNAs, each of which has at most 2,500 secondary structures, no such relation was found. Even worse, for the set of 1000 RNAs, the correlation between Monte Carlo MFPT and Gillespie MFTP times the expected number of neighbors was found to be lower than the correlation without its consideration. This result suggests that number of trajectory steps necessary to reach the minimum free energy structure may be too small to see the asymptotic relation expected by Theorem 11. We conclude that RNA secondary structure folding may occur faster than the time scale required for Theorem 1, a type of Levinthal paradox [31] which suggests that folding pathways could be encoded in RNA sequences, or that RNA secondary structure formation could follow either a kinetic funnel model [8] or a kinetic hub model [7.

Next, we argue that macromolecular folding kinetics are better captured by Gillespie's Algorithm 4, than Monte Carlo Algorithms 1,2,3. For all but pathological RNA sequences, it is the case that detailed balance $\pi_{x} \cdot p_{x, y}=\pi_{y} \cdot p_{y, x}$ does not hold for the Boltzmann distribution $\pi$, as shown by considering $x$ to be the empty structure and $y$ to be a structure containing exactly one base pair. Since the Boltzmann distribution is not necessarily the stationary distribution, this argument does not imply that detailed balance does not hold; however in Section 4.4 the (non-Boltzmannian) stationary distribution is computed for a tiny RNA sequence, for which it is shown that detailed balance does not hold.

Since the stationary distribution for the Markov chain underlying each of the Monte Carlo Algorithms $1,2,3$ is not necessarily the Boltzmann distribution, one might instead consider modified versions of these algorithms defined as follows. Algorithm $1^{\ddagger}$ is obtained from Algorithm $1^{\dagger}$ by replacing the expression in line 9 by

or equivalently by

$$
\frac{\pi_{y}}{\pi_{x}} \cdot \frac{N_{x}}{N_{y}}
$$

$$
\exp \left(\frac{-(E(y)-E(x))}{R T}\right) \cdot \frac{N_{x}}{N_{y}} .
$$

Similarly, Algorithm $2^{\ddagger}$ [resp. $3^{\ddagger}$ ] is obtained from Algorithm $2^{\dagger}$ [resp. $\left.3^{\dagger}\right]$ by replacing the expression in line 9 by

$$
\frac{1}{N_{x}} \cdot \min \left(1, \frac{\pi_{y}}{\pi_{x}} \cdot \frac{N_{x}}{N_{y}}\right)
$$

or equivalently

$$
\frac{1}{N_{x}} \cdot \min \left(1, \exp \left(\frac{-(E(y)-E(x))}{R T}\right) \cdot \frac{N_{x}}{N_{y}}\right) .
$$

This ensures that the new Markov chain underlying each of the Algorithms $1^{\ddagger}, 2^{\ddagger}, 3^{\ddagger}$ is Markov chain $\mathbb{M}_{H}$, whose transition probability matrix $P_{H}=\left(p_{x, y}^{H}\right)$ is defined by Hastings' trick, i.e.

$$
p_{x, y}^{H}=\frac{1}{N_{x}} \min \left(1, \exp (-(E(y)-E(x)) / R T) \cdot N_{x} / N_{y}\right)
$$

However, the embedded matrix (jump matrix) for $M_{H}$ would then be different for the Markov process $\mathbb{M}_{2}$ underlying the Gillespie algorithm. Although we would have ensured that the stationary distribution for

\footnotetext{
${ }^{4} \mathrm{ME}$ stands for the Master Equation, meaning the computation of the time-dependent population occupancy vector $\mathbf{p}(t)$ by solution of the master equation $\frac{d \mathbf{p}(t)}{d t}=\mathbf{p}(t) \cdot Q$, where $Q$ is the rate matrix. MC stands for Monte Carlo simulation, and MMC stands for Map-based Monte Carlo simulation, a method inspired by probabilistic road map methods from robotics, where a constant $k$ many closest neighboring secondary structures are selected for each RNA secondary structure from a sampled collection of modest size. Subsequently Monte Carlo simulation is applied to the resulting network, which is much smaller than the network of all secondary structures. The correctness of the authors' assertion is due uniquely to the fact that the roadmap sampling network is $k$-regular.
} 
each of the Algorithms $1^{\ddagger}, 2^{\ddagger}, 3^{\ddagger}$, and 4 is the Boltzmann distribution, we would no longer have trajectory times related as in Theorem 1 .

By Theorem 1, either the Monte Carlo algorithm or the Gillespie algorithm may be used for statistical analysis of trajectories (frequency of visitation of states, etc.). However in the context of RNA secondary structure folding time to the minimum free energy structure, the Boltzmann distribution is the stationary distribution only for the Gillespie Algorithm 4 and for the Monte Carlo Algorithms 2,3 modified by Hastings' trick using transition probabilities from equation 20 . Moreover, the minimum free energy structure is targeted only if the Boltzmann distribution is the stationary distribution, where by targeted, we mean that the MFE structure has the highest probability, and that $\lim _{n \rightarrow \infty} \operatorname{Pr}\left[X_{n}=x\right]=\pi_{x}$, where $X_{n}$ is the random variable for the secondary structure at step $n$ and $\pi_{x}$ is the Boltzmann probability of structure $x$. However, there is a serious computational cost for the Hastings-modified Monte Carlo algorithm; indeed, determination of the update to state $x$ requires the computation of free energies of all neighbors of not only current state $x$, but as well of all neighbors in $N_{y}$ of all states $y \in N_{x}$. For repeated RNA kinetics simulations, this cost could be prohibitive.

We argue that the Gillespie algorithm should be used for RNA secondary structure folding kinetics, and that specifically one should compute the population occupancy vector $\mathbf{p}(t)$ determined by solution of the matrix differential equation $\frac{d \mathbf{p}(t)}{d t}=\mathbf{p}(t) \cdot Q$, for rate matrix $Q$ as in [58, 56, rather than the mean first passage time by use of the fundamental matrix or by matrix inversion [35] as done for Markov state models in [7, 25]. For synthetic design of RNA molecules [57, 13, we advocate fast MFPT computation using coarse-grained models [52, 43, to select design candidates for subsequent scrutiny by more accurate methods such as KFOLD.

\section{Acknowledgements}

This research was begun during a visit to California Institute of Technology, Free University of Berlin and the Max Planck Institute for Molecular Genetics. For discussions, P.C. would like to thank Frank Noé, Knut Reinert, Martin Vingron and Marcus Weber (Berlin) and Niles Pierce, Erik Winfree (Pasadena). The research was funded by a Guggenheim Fellowship, Deutscher Akademischer Austauschdienst (DAAD), and by National Science Foundation grant DBI-1262439. Any opinions, findings, and conclusions or recommendations expressed in this material are those of the authors and do not necessarily reflect the views of the National Science Foundation. 


\section{Algorithm 1 (Discrete-time time-driven MC algorithm for MFPT)}

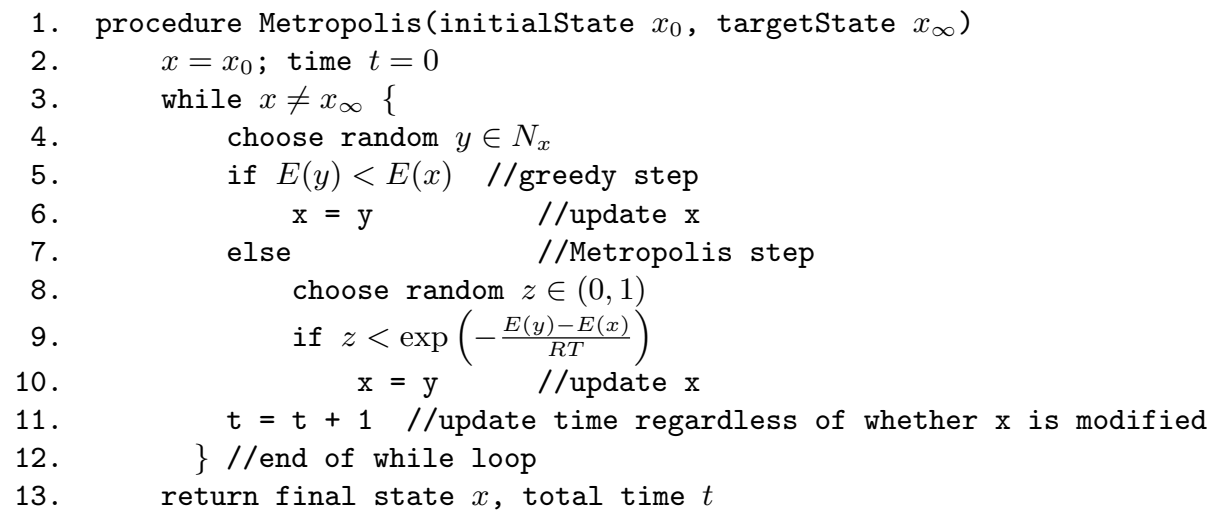

Figure 1: Discrete-time time-driven Monte Carlo (MC) algorithm to estimate mean first passage time for a given Markov chain, whose transition probabilities are given by equation (2). Since the Boltzmann probabilities are defined by $p_{x}^{*}=\exp (-E(x) / R T) / Z, p_{y}^{*}=\exp (-E(y) / R T) / Z$, it follows that $p_{y}^{*} / p_{x}^{*}=\exp (-(E(y)-E(x)) / R T)$, so that the probability that state $x$ is modified to $y$ in lines 5 -10 is precisely $p_{x, y}=1 / N_{x} \cdot \min (1, \exp (-(E(y)-E(x)) / R T))$. 


\section{Algorithm 2 (Discrete-time event-driven MC algorithm for MFPT)}

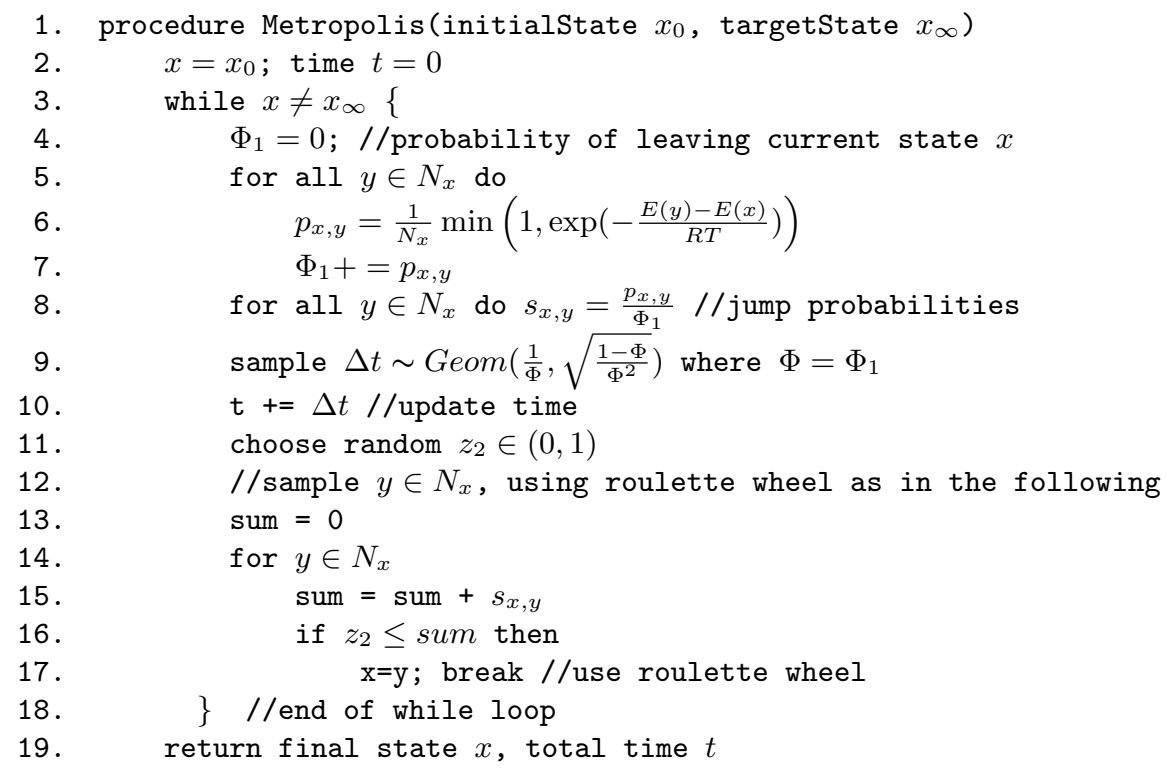

Figure 2: Discrete-time event-driven Monte Carlo (MC) algorithm to estimate mean first passage time for a given Markov chain, whose transition probabilities are given by equation (2). Since the probability that state $x$ is modified to $y$ in lines $5-10$ of Algorithm 1 equals $p_{x, y}=1 / N_{x} \cdot \min (1, \exp (-(E(y)-E(x)) / R T))$, it follows that the probability that $x$ will be modified is $\sum_{z \in N_{x}} p_{x, z}$ which equals $1-p_{x, x}$. The probability that $x$ will be modified in the $k$ th iteration of the while loop of Algorithm 1 (but not before) is exactly $p_{x, x}^{k-1} \cdot\left(1-p_{x, x}\right)$; i.e. equivalent to the probability that the heads is obtained in the $k$ th coin flip (but not before) for a coin whose heads probability is $\left(1-p_{x, x}\right)$, given as $\Phi$ in line 7 of Algorithm 2 . It follows that the number of steps $\Delta t$ before the state $x$ is modified is geometrically distributed with parameter $\Phi=\left(1-p_{x, x}\right)$; since the mean [resp. standard deviation] for a geometrically distributed random variable with parameter $\Phi$ is $\frac{1}{\Phi}$ [resp. $\left.\sqrt{\frac{1-\Phi}{\Phi^{2}}}\right]$ we denote the distribution by $\operatorname{Geom}\left(\frac{1}{\Phi}, \sqrt{\frac{1-\Phi}{\Phi^{2}}}\right)$. Time increments $\Delta t$ are sampled from this geometric distribution, hence the expected time increment in line 9 of Algorithm 2 is identical to the expected time increment before state $x$ is modified in lines 4-10 of Algorithm 1, so both algorithms are equivalent. 


\section{Algorithm 3 (Continuous-time event-driven MC algorithm for MFPT)}

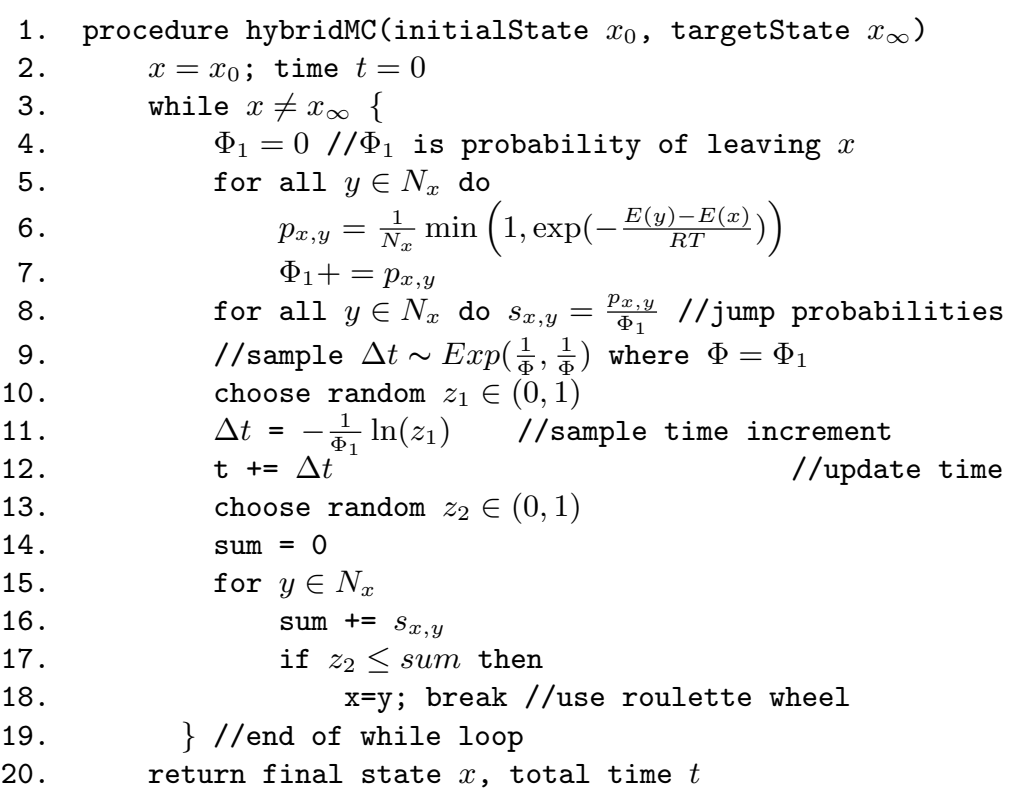

Figure 3: Continuous-time event-driven Monte Carlo (MC) algorithm, to estimate mean first passage time for a given Markov chain, whose transition probabilities are given by equation (2). The geometically distibuted time increment $\Delta t$ from line 9 of Algorithm 2 is replaced in line 9 of the current algorithm by the exponentially distributed time increment having the same expectation $\frac{1}{\Phi}$. As the standard deviatioin of the exponential distribution with parameter $1 / \Phi$ is also $1 / \Phi$, we denote this distribution by $\operatorname{Exp}\left(\frac{1}{\Phi}, \frac{1}{\Phi}\right)$ - this allows the reader to immediately see that the time increment $\Delta t$ in line 9 of both algorithms has the same mean and approximately the same standard deviation (especially if $\Phi$ is small). 


\section{Algorithm 4 (Gillespie's algorithm for MFPT)}

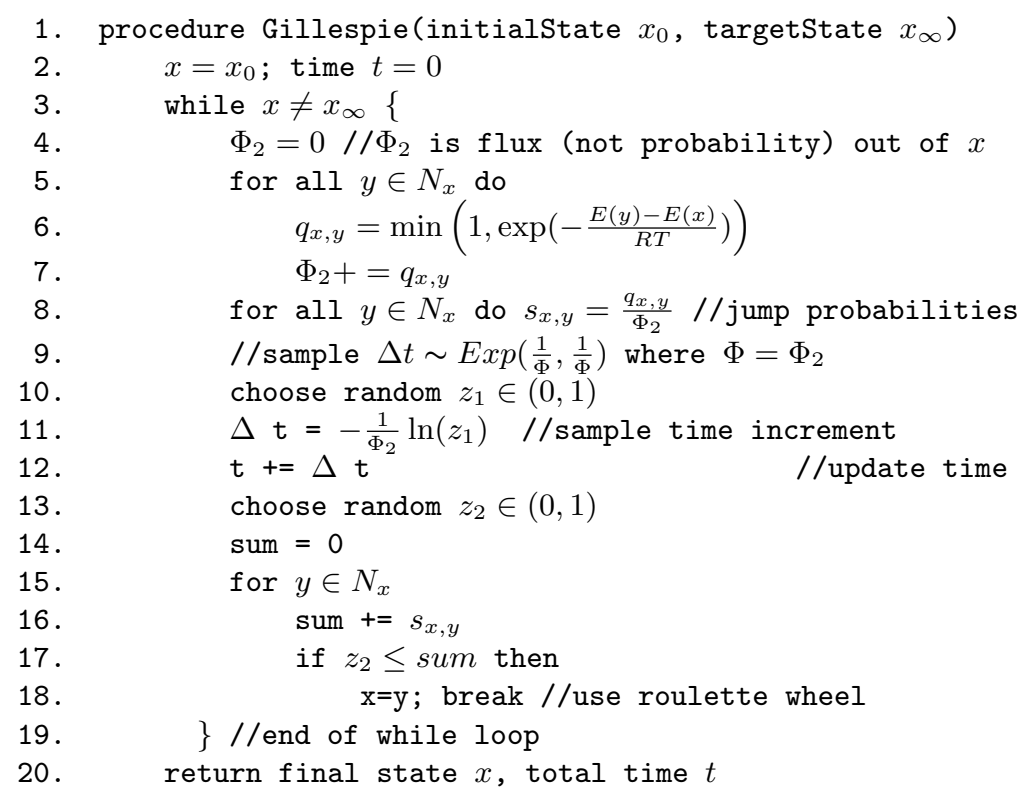

Figure 4: Continuous-time event-driven Gillespie algorithm, to estimate mean first passage time for a given Markov process, whose transition rates are given by equation (4). The only difference between this algorithm and Algorithm 3 is that in line 6, the transition rate $q_{x, y}$ is missing the factor $1 / N_{x}$ present in transition probability $p_{x, y}$, and so the value $\Phi$ from line 9 is smaller in the current algorithm than in Algorithm 3 by the factor $N_{x}$. It immediately follows that Algorithms 3 and 4 are identical in a regular network where $N_{x}=N$ for all states $x$; moreover, it is suggestive (but incorrect for MFPT) that the values $\Phi$ from line 9 of Algorithms 3 and 4 might be related by the Boltzmann expected number $\left\langle N_{x}\right\rangle$ of neighbors. 

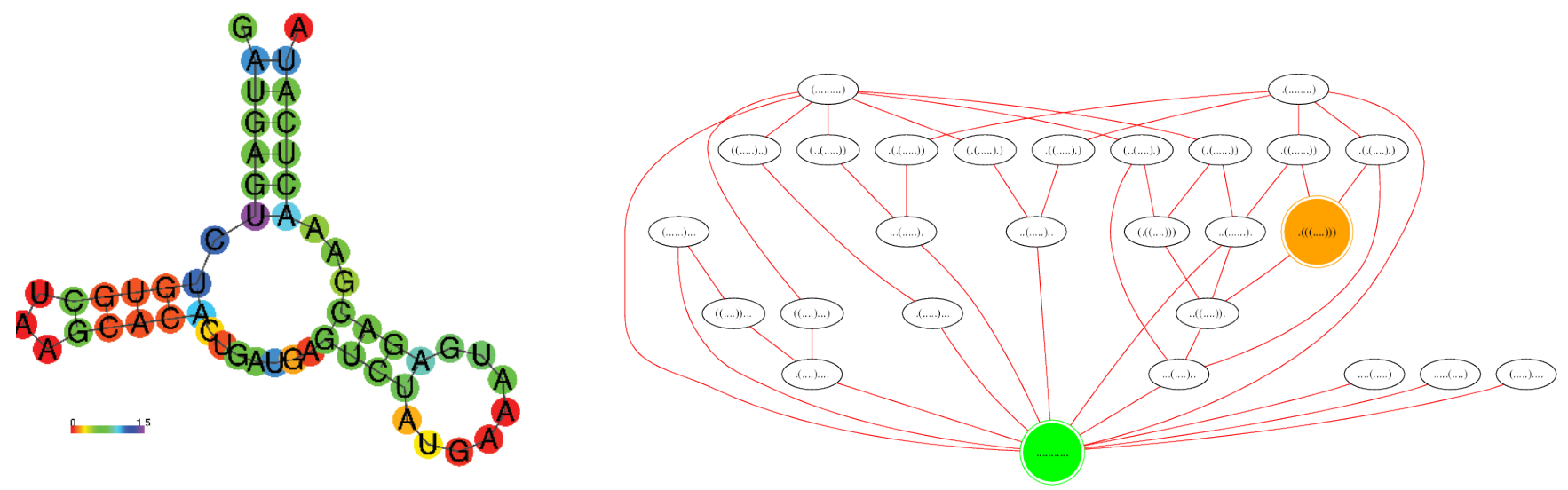

Figure 5: (Left) Minimum free energy secondary (MFE) structure of the 54 nt Peach Latent Mosaic Viroid (PLMVd) AJ005312.1/282-335, colored by positional entropy - see [26] for the definition of positional entropy. The MFE structure was computed by RNAfold using Vienna RNA Package 1.8.5, employing the Turner 1999 free energy parameters. This structure is identical to the consensus structure from the Rfam database 20, while the structure produced by Vienna RNA Package 2.1.9, employing the Turner 2004 free energy parameters consists of two external stem-loops. This example shows the sometimes dramatic difference in MFE structure computation due solely to slightly different free energy parameters [50. (Right) Network of secondary structures for the 11-nt RNA sequence GGCCGGCCGGC, where edges are indicated between every two structures whose base pair distance is 1 (i.e. move set $M S_{1}$ ). The expected degree for this network having 27 nodes and 42 edges is 3.111111 with respect to the uniform distribution and 3.074169 with respect to the Boltzmann distribution. The MFE structure of GCGCGCGCGCG, depicted in the orange circle, has free energy $-2.6 \mathrm{kcal} / \mathrm{mol}$. Mean first passage time for secondary structure folding is the average first passage time from the empty initial structure (green circle) to the MFE structure (orange circle), where edge probabilities [rates] for the Monte Carlo [resp. Gillespie] algorithm is given by equation (2) [resp. equation (4)]. 


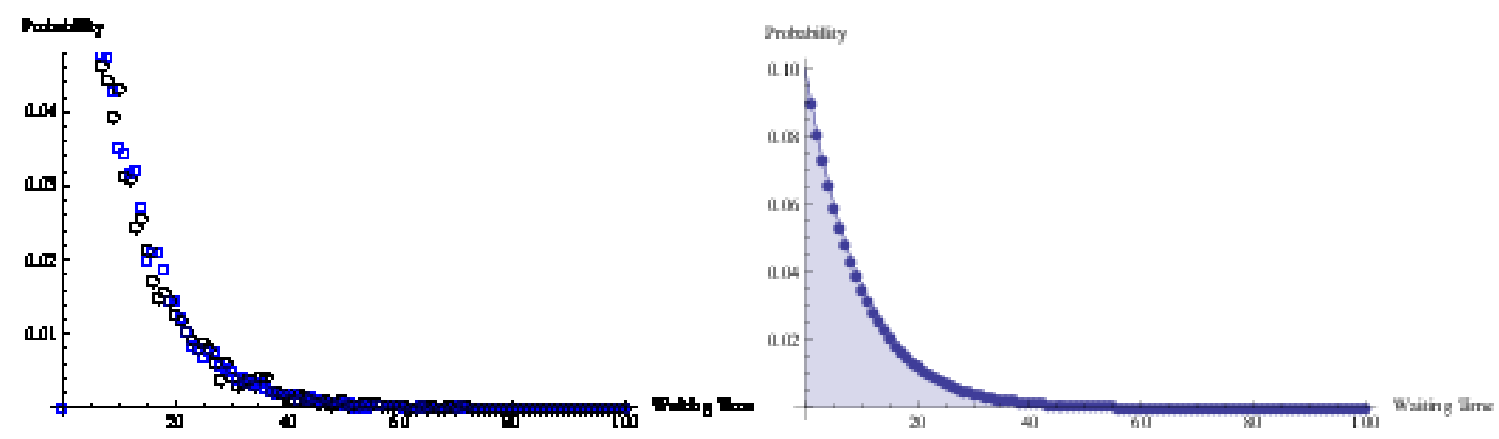

Figure 6: (Left) Superimposition of histograms for the geometric and exponential distributions. In each case, using the method described in the text, 10,000 points were sampled from the geometric (blue squares) [resp. exponential (black circles)] distribution having mean 10 and standard deviation $\sqrt{90} \approx 9.4868$ [resp. mean 10]. For the geometric distribution, this corresponds to the waiting time before obtaining a heads, where the probability of flipping a heads is 0.1 . For the 10,000 points sampled from the geometric distribution, the sample mean is 9.995400 and the sample standard deviation is 9.413309 . For the 10,000 points sampled from the exponential distribution, the sample mean is 9.989983 and the sample standard deviation is 9.950308. (Right) Superimposition of the probability [resp. probability density] of the geometric [resp. exponential] distribution having mean of 10, as computed by Mathematica. Graphics produced with Mathematica.
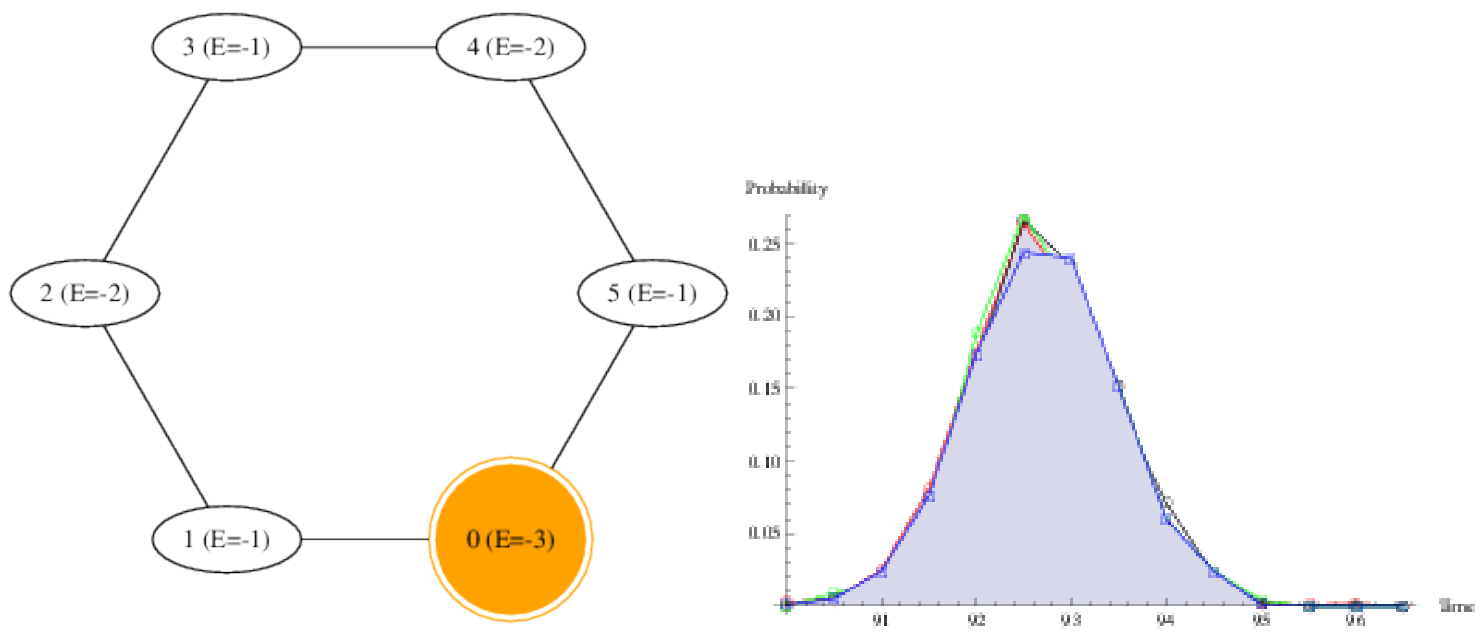

Figure 7: (Left) Topology of Markov chain/process from Example 1, consisting of $2 n$ states $0,1, \ldots, 2 n-1$, each having exactly two neighbors. State 0 has energy -3 , non-zero even [resp. odd] states have energy -2 [resp. -1]. The figure illustrates the topology for $n=3$, Example 1 in the text considers $n=10$. (Right) Superimposed histograms for Monte Carlo and Gillespie first passage times for Example 1, taken over 1000 computational experiments, each experiment consisting of taking the average first passage time in 10,000 repetitions of each of the following algorithms: (1) time-driven Monte Carlo algorithm (blue squares with filled area), (2) event-driven Monte Carlo algorithm with geometrically distributed waiting times (black circles), (3) event-driven Monte Carlo algorithm with exponentially distributed waiting times (green squares), (4) Gillespie's algorithm (red circles). Histograms for a single experiment for each algorithm, consisting of 10,000 separate runs, resembled an exponential distribution, of course, but the averages of each experiment are approximately normally distributed (central limit theorem). 

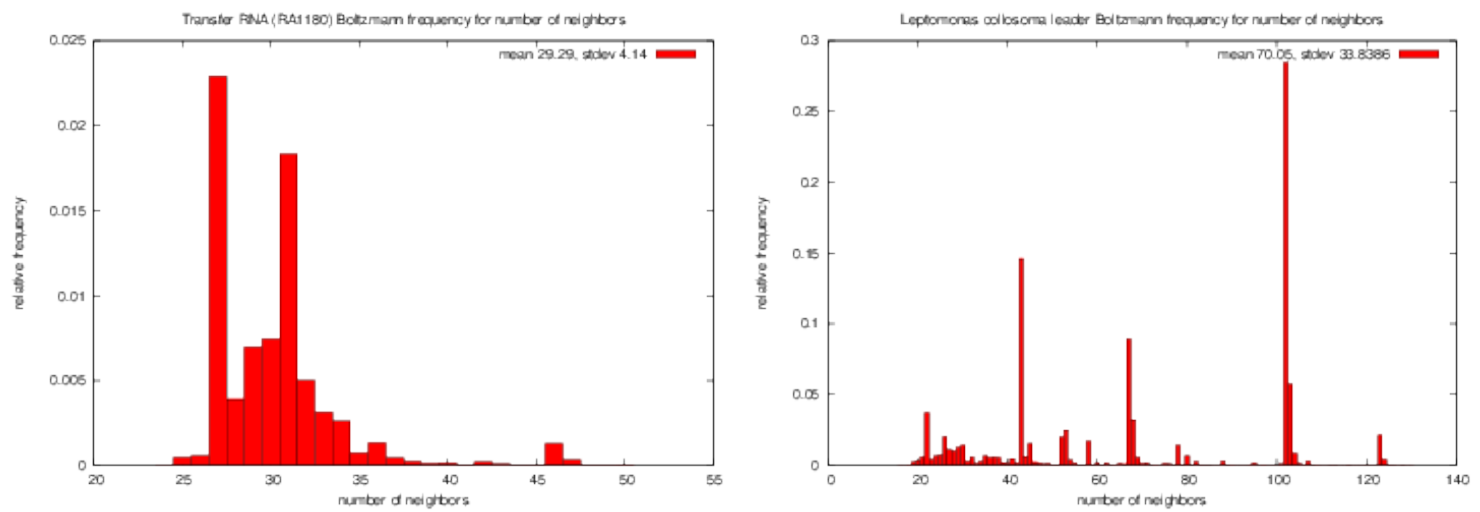

Figure 8: Distribution of the Boltzmann-weighted number of neighbors, where the number of neighbors of structure $s$ is the number of structures $t$ obtained from $s$ by removal or addition of a single base pair. Relative frequency at position $x$ is defined as the sum, taken over all sampled structures $s$ of degree $x$, of the Boltzmann probability $\exp (-E(s) / R T) / Z^{*}$, where $Z^{*}$ is the sum of Boltzmann factors of all sampled structures. This value approximates the true Boltzmann probability $\exp (-E(s) / R T) / Z$, where the partition function $Z$ is the sum of Boltzmann factors of all structures. (Left) Distribution of the number of neighbors for transfer RNA (RA1180 from tRNAdb 2009 [29]), having expected number of structures $\langle N\rangle=29.29 \pm 4.14$. Using RNAsubopt -e 12 [33, all structures were generated, whose free energy is within $12 \mathrm{kcal} / \mathrm{mol}$ of the minimum free energy (110,572 structures), and the ratio $Z^{*} / Z$ of the sum of corresponding Boltzmann factors divided by the partition function is 0.9990. (Right) Distribution of the number of neighbors for spliced leader RNA, an RNA conformational switch, from the trypanosome Leptomonas collosoma, having expected number of structures $\langle N\rangle=70.05 \pm 33.84$. A total of 57,803 structures were generated using RNAsubopt -e 10, and the ratio $Z^{*} / Z$ of the sum of corresponding Boltzmann factors divided by the partition function is 0.9992 . 

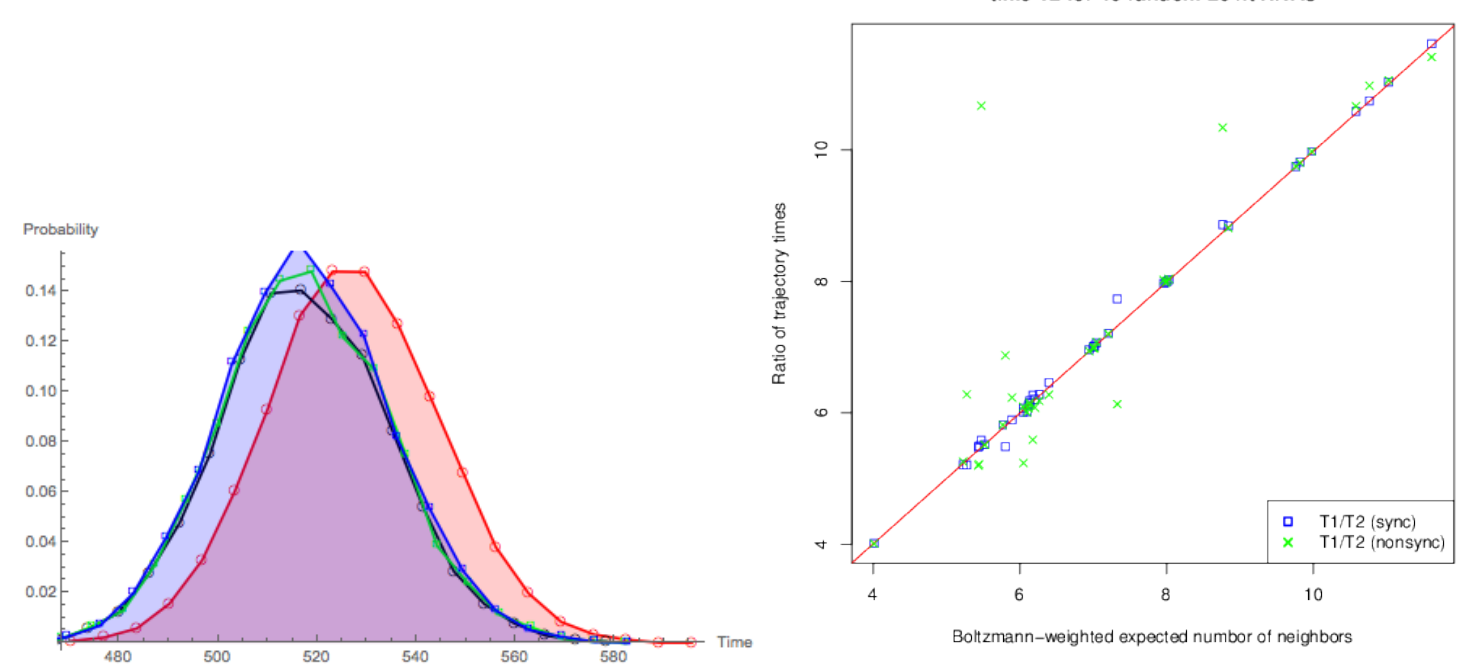

Figure 9: (Left) Superimposed histograms for Monte Carlo and Gillespie first passage times for Example 2 taken over 1000 computational experiments, each experiment consisting of taking the average first passage time in 10,000 repetitions of each of the algorithms: (1) time-driven Monte Carlo algorithm (blue squares with filled area), (2) event-driven Monte Carlo algorithm with geometrically distributed waiting times (black circles), (3) event-driven Monte Carlo algorithm with exponentially distributed waiting times (green squares), (4) Gillespie's algorithm (red circles). The computations were performed exactly as in Figure 7 - the topology of Example 2 differs from that of Example 1 only in that there is no edge between the minimum energy state 0 and the last state $2 n-1$, i.e. state 19 in Example 2. (Right) Ratio $\frac{\tau_{1}^{K}}{\tau_{2}^{K}}$ of trajectory time for $K$ steps $(K=1$ million) in Monte Carlo and Gillespie algorithms, plotted as a function of the expected number of neighbors (expected degree) for 40 randomly generated $20 \mathrm{nt}$ RNAs. This figure illustrates Theorem 1, which states that the ratio $\frac{E\left(\tau_{1}^{K}\right)}{E\left(\tau_{2}^{K}\right)}$ of expected trajectory time for $K$-step trajectories using Monte Carlo Algorithm $3^{\dagger}$ over the expected trajectory time of $K$-step trajectories using Gillespie's Algorithm $4^{\dagger}$ equals the expected degree of the network of RNA secondary structures, as $K$ increases to infinity. Both synchronized and unsynchronized trajectories are plotted. 

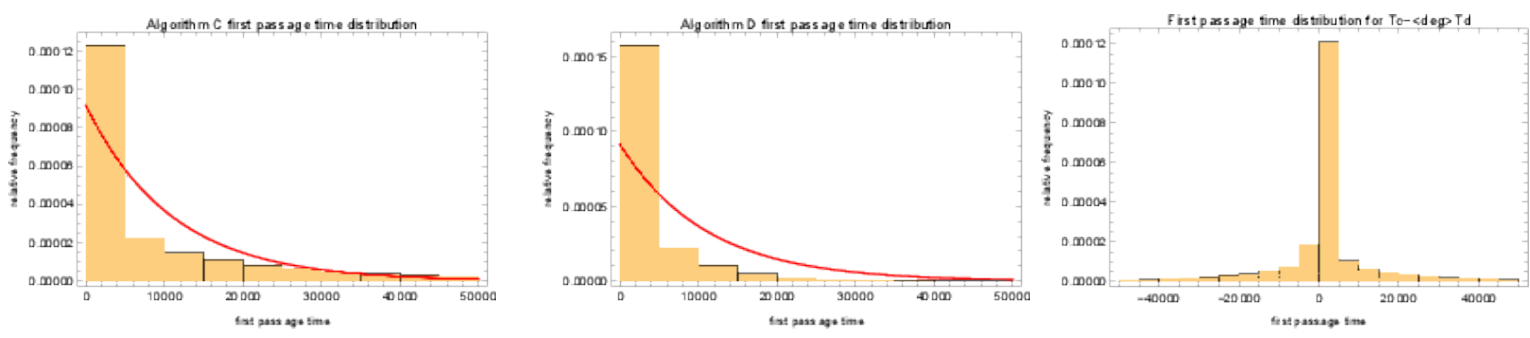

Figure 10: Relative histograms for first passage times for the continuous-time, event-driven Monte Carlo algorithm and the Gillespie algorithm. (a) Probability distribution for Algorithm 3 trajectory time $T_{c}$ from the empty initial structure to the MFE structure, with mean of 10953.60, standard deviation of 17765.47, maximum of $221,692.29$, and minimum of 0.41 . Red line shows the exponential distribution with parameter $\lambda=\frac{1}{10953.60} \approx 9.13 \times 10^{-5}$. (b) Probability distribution for Algorithm 4 trajectory time $T_{d}$ from the empty initial structure to the MFE structure, with mean of 3192.31, standard deviation of 5654.83, maximum of 72,473.93, and minimum of 0.075 . Red line shows the exponential distribution with parameter $\lambda=$ $\frac{1}{3192.31} \approx 3.13 \times 10^{-4}$ (c) Probability distribution for time $T_{c}-\langle N\rangle \cdot T_{d}$, where $\langle N\rangle=3.031162 \approx 3.03$ is the expected number of neighbors for the network of all 62 secondary structures for the 10-nt sequence GGGGGCCCCC. This distribution has mean of 1277.20, standard deviation of 18,379.89, maximum of 204, 747.05, and minimum of $-199,785.20$. See text for a proof that with overwhelming statistical significance we have the strict inequalities $\langle N\rangle_{b} \cdot T_{d}\left\langle T_{c}\left\langle\langle N\rangle_{u} \cdot T_{d}\right.\right.$, where $\langle N\rangle_{b}$ [resp. $\langle N\rangle_{u}$ ] denotes the Boltzmann [resp. uniform] expected number of neighbors. 


\section{References}

[1] V. I. Abkevich, A. M. Gutin, and E. I. Shakhnovich. How the first biopolymers could have evolved. Proc. Natl. Acad. Sci. U.S.A., 93(2):839-844, January 1996.

[2] V. I. Abkevich, A. M. Gutin, and E. I. Shakhnovich. Computer simulations of prebiotic evolution. Pac Symp Biocomput., $0(\mathrm{O}): \mathrm{O}, 1997$.

[3] C. B. Anfinsen. Principles that govern the folding of protein chains. Science, 181:223-230, 1973.

[4] I. Aviram, I. Veltman, A. Churkin, and D. Barash. Efficient procedures for the numerical simulation of mid-size RNA kinetics. Algorithms. Mol. Biol., 7(1):24, 2012.

[5] G. R. Bowman. A tutorial on building markov state models with MSMBuilder and coarse-graining them with BACE. Methods Mol. Biol., 1084:141-158, 2014.

[6] G. R. Bowman, X. Huang, and V. S. Pande. Using generalized ensemble simulations and Markov state models to identify conformational states. Methods, 49(2):197-201, October 2009.

[7] G. R. Bowman and V. S. Pande. Protein folded states are kinetic hubs. Proc. Natl. Acad. Sci. U.S.A., 107(24):10890-10895, June 2010.

[8] J. D. Bryngelson, J. N. Onuchic, N. D. Socci, and P. G. Wolynes. Funnels, pathways, and the energy landscape of protein folding: a synthesis. Proteins., 21(3):167-195, March 1995.

[9] H. M. Choi, V. A. Beck, and N. A. Pierce. Multiplexed in situ hybridization using hybridization chain reaction. Zebrafish., 11(5):488-489, October 2014.

[10] P Clote. Expected degree for RNA secondary structure networks. J Comp Chem, 36(2):103-17, Jan 2015.

[11] P. Clote and R. Backofen. Computational Molecular Biology: An Introduction. John Wiley \& Sons, 2000.286 pages.

[12] P. Clote and A. Bayegan. Network Properties of the Ensemble of RNA Structures. PLoS. One., 10(10):e0139476, 2015.

[13] I. Dotu, J. A. Garcia-Martin, B. L. Slinger, V. Mechery, M. M. Meyer, and P. Clote. Complete RNA inverse folding: computational design of functional hammerhead ribozymes. Nucleic. Acids. Res., 42(18):11752-11762, February 2015.

[14] E. C. Dykeman. An implementation of the Gillespie algorithm for RNA kinetics with logarithmic time update. Nucleic. Acids. Res., 43(12):5708-5715, July 2015.

[15] A. Esmaili-Taheri and M. Ganjtabesh. ERD: a fast and reliable tool for RNA design including constraints. $B M C$. Bioinformatics, 16(1):20, January 2015.

[16] W. Feller. An Introduction to Probability Theory and its Applications, volume 1. John Wiley and Sons, New York, 3rd edition, 1968.

[17] C. Flamm, W. Fontana, I.L. Hofacker, and P. Schuster. RNA folding at elementary step resolution. RNA, 6:325-338, 2000.

[18] E. Fusy and P. Clote. Combinatorics of locally optimal RNA secondary structures. J Math Biol., 0(O):O, December 2012.

[19] J.A. Garcia-Martin, P. Clote, and I. Dotu. RNAiFold: A constraint programming algorithm for RNA inverse folding and molecular design. J Bioinform Comput Biol., 11(2):1350001, 2013. DOI: 10.1142/S0219720013500017.

[20] P. P. Gardner, J. Daub, J. Tate, B. L. Moore, I. H. Osuch, S. Griffiths-Jones, R. D. Finn, E. P. Nawrocki, D. L. Kolbe, S. R. Eddy, and A. Bateman. Rfam: Wikipedia, clans and the "decimal" release. Nucleic. Acids. Res., 39(Database):D141-D145, January 2011.

[21] M. Geis, C. Flamm, M. T. Wolfinger, A. Tanzer, I. L. Hofacker, M. Middendorf, C. Mandl, P. F. Stadler, and C. Thurner. Folding kinetics of large RNAs. J. Mol. Biol., 379(1):160-173, May 2008.

[22] D.T. Gillespie. A general method for numerically simulating the stochastic time evolution of coupled chemical reactions. J Comp Phys, 22(403):403-434, 1976.

[23] M. P. Harrigan, M. M. Sultan, C. X. Hernandez, B. E. Husic, P. Eastman, C. R. Schwantes, K. A. Beauchamp, R. T. McGibbon, and V. S. Pande. MSMBuilder: Statistical Models for Biomolecular Dynamics. Biophys. J., 112(1):10-15, January 2017.

[24] W.K. Hastings. Monte carlo sampling methods using markov chains and their applications. Biometrika, 57(1):97-109, April 1970.

[25] X. Huang, Y. Yao, G. R. Bowman, J. Sun, L. J. Guibas, G. Carlsson, and V. S. Pande. Constructing multi-resolution Markov State Models (MSMs) to elucidate RNA hairpin folding mechanisms. Pac Symp Biocomput., 0(O):O, 2010:228-39.

[26] M. Huynen, R. Gutell, and D. Konings. Assessing the reliability of RNA folding using statistical mechanics. J. Mol. Biol., 267(5):1104-1112, April 1997.

[27] Jr. I. Tinoco and M. Schmitz. Thermodynamics of formation of secondary structure in nucleic acids. In E. Di Cera, editor, Thermodynamics in Biology, pages 131-176. Oxford University Press, 2000.

[28] H. Isambert and E. D. Siggia. Modeling RNA folding paths with pseudoknots: application to hepatitis delta virus ribozyme. Proc. Natl. Acad. Sci. U.S.A., 97(12):6515-6520, June 2000.

[29] F. Juhling, M. Morl, R. K. Hartmann, M. Sprinzl, P. F. Stadler, and J. Putz. tRNAdb 2009: compilation of tRNA sequences and tRNA genes. Nucleic. Acids. Res., 37(Database):D159-D162, January 2009. 
[30] Konstantin Klemm, Christoph Flamm, and Peter F. Stadler. Funnels in energy landscapes. The European Physical Journal $B$, pages 387-391, 2008.

[31] C. Levinthal. Are there pathways for protein folding? Journal de Chimie Physique, 65:44-45, 1968.

[32] R. M. Levy, W. Dai, N. J. Deng, and D. E. Makarov. How long does it take to equilibrate the unfolded state of a protein? Protein. Sci., 22(11):1459-1465, November 2013.

[33] R. Lorenz, S. H. Bernhart, C. Höner zu Siederdissen, H. Tafer, C. Flamm, P. F. Stadler, and I. L. Hofacker. Viennarna Package 2.0. Algorithms. Mol. Biol., 6:26, 2011.

[34] N. Metropolis, A.W. Rosenbluth, M.N. Rosenbluth, A.H. Teller, and E. Teller. Equation of state calculations by fast computing machines. J. Chem. Phys., 21:1087-1092, 1953.

[35] C.D. Meyer. The role of the group inverse in the theory of finite Markov chains. SIAM Rev., 17(46):443-464, 1975.

[36] V. Moulton, M. Zuker, M. Steel, R. Pointon, and D. Penny. Metrics on RNA secondary structures. Journal of Computational Biology, 7:277-292, 2000.

[37] E. P. Nawrocki, S. W. Burge, A. Bateman, J. Daub, R. Y. Eberhardt, S. R. Eddy, E. W. Floden, P. P. Gardner, T. A. Jones, J. Tate, and R. D. Finn. Rfam 12.0: updates to the RNA families database. Nucleic. Acids. Res., 43(Database):D130-D137, January 2015.

[38] R. Nussinov and A. B. Jacobson. Fast algorithm for predicting the secondary structure of single stranded RNA. Proceedings of the National Academy of Sciences, USA, 77(11):6309-6313, 1980.

[39] V. S. Pande, K. Beauchamp, and G. R. Bowman. Everything you wanted to know about Markov State Models but were afraid to ask. Methods, 52(1):99-105, September 2010.

[40] A. Sali, E. Shakhnovich, and M. Karplus. How does a protein fold? Nature, 369(6477):248-251, May 1994.

[41] A. Šali, E. Shakhnovich, and M. Karplus. Kinetics of protein folding: A lattice model study of the requirements for folding to the native state. Journal of Molecular Biology, 235:1614-1636, 1994.

[42] M. Schmitz and G. Steger. Description of RNA folding by "simulated annealing". J. Mol. Biol., 255(1):254-266, January 1996.

[43] E. Senter and P. Clote. Fast, approximate kinetics of RNA folding. J. Comput. Biol., 22(2):124-144, February 2015.

[44] E. Senter, I. Dotu, and P. Clote. RNA folding pathways and kinetics using 2D energy landscapes. J. Math. Biol., 70(1-2):173-196, January 2015.

[45] E. Senter, S. Sheikh, I. Dotu, Y. Ponty, and P. Clote. Using the fast fourier transform to accelerate the computational search for RNA conformational switches. PLoS. One., 7(12):e50506, 2012.

[46] P. R. Stein and M. S. Waterman. On some new sequences generalizing the Catalan and Motzkin numbers. Discrete Mathematics, 26:261-272, 1978.

[47] W.C. Swope, J.W. Pitera, and F. Suits. Describing protein folding kinetics by molecular dynamics simulations. 1. Theory. J Phys Chem B, 108(21):6571-6581, 2010:228-39.

[48] A. Taneda. MODENA: a multi-objective RNA inverse folding. Adv. Appl. Bioinform. Chem., 4:1-12, 2011.

[49] X. Tang, S. Thomas, L. Tapia, D. P. Giedroc, and N. M. Amato. Simulating RNA folding kinetics on approximated energy landscapes. J. Mol. Biol., 381(4):1055-1067, September 2008.

[50] D. H. Turner and D. H. Mathews. NNDB: the nearest neighbor parameter database for predicting stability of nucleic acid secondary structure. Nucleic. Acids. Res., 38(Database):D280-D282, January 2010.

[51] J. K. Weber and V. S. Pande. Characterization and rapid sampling of protein folding Markov state model topologies. J. Chem. Theory Comput., 7(10):3405-3411, October 2011.

[52] M. Wolfinger, W.A. Svrcek-Seiler1, C. Flamm, and P.F. Stadler. Efficient computation of RNA folding dynamics. J Phys. A: Math. Gen., 37:4731-4741, 2004.

[53] Michael T. Wolfinger, W. Andreas Svrcek-Seiler, Christoph Flamm, Ivo L. Hofacker, and Peter F. Stadler. Efficient folding dynamics of RNA secondary structures. J. Phys. A: Math. Gen., 37:4731-4741, 2004.

[54] S. Wuchty, W. Fontana, I. L. Hofacker, and P. Schuster. Complete suboptimal folding of RNA and the stability of secondary structures. Biopolymers, 49:145-165, 1999.

[55] T. Xinyu, S. Thomas, L. Tapia, and N.M. Amato. Tools for simulating and analyzing RNA folding kinetics. In Proceedings: RResearch in Computational Molecular Biology, 11th Annual International Conference (RECOMB), pages 268-282. ACM Press, April 2007. San Francisco, CA.

[56] X. Xu, T. Yu, and S. J. Chen. Understanding the kinetic mechanism of RNA single base pair formation. Proc. Natl. Acad. Sci. U.S.A., 113(1):116-121, January 2016.

[57] J. N. Zadeh, B. R. Wolfe, and N. A. Pierce. Nucleic acid sequence design via efficient ensemble defect optimization. J. Comput. Chem., 32(3):439-452, February 2011.

[58] W. Zhang and S. J. Chen. RNA hairpin-folding kinetics. Proc. Natl. Acad. Sci. U.S.A., 99(4):1931-1936, February 2002.

[59] M. Zuker and D. Sankoff. RNA secondary structures and their prediction. Bulletin of Biology, 46(4):591-621, 1984.

[60] M. Zuker and P. Stiegler. Optimal computer folding of large RNA sequences using thermodynamics and auxiliary information. Nucleic Acids Res., 9:133-148, 1981. 


\section{Appendix}

\section{A Markov chain of RNA secondary structures and detailed bal- ance}

In this section, we prove that detailed balance does not hold for the Markov chain $\mathcal{M}_{1}$ of secondary structures for an RNA sequence, where transition probabilities are defined by equation (2). The failure of detailed balance is because for almost any RNA sequence, there are secondary structures $x, y$ that are neighbors of each other, but number $N(x)$ of neighbors of $x$ does not equal the number $N(y)$ of neighbors of $y$. If every secondary structure of a given RNA sequence has the same number of neighbors, then it is easy to show that the Boltzmann distribution $\left(\pi_{1}, \ldots, \pi_{n}\right)$ is the stationary distribution, and a small calculation then shows that detailed balance holds. If different structures have different numbers of neighbors, then the Boltzmann distribution is not in general the stationary distribution. To produce an example where detailed balance fails, we must compute the stationary distribution.

For convenience in following the computation, we first present a simple example using the Nussinov energy model, in which the energy $E(s)$ of a structure is defined to be -1 multiplied by the number of base pairs. Historically, the Nussinov algorithm and energy model [38] predate the Zuker algorithm 60] and Turner energy model [50].

Example 3 (Detailed balance does not hold for Nussinov energy model) Consider the 6-nt RNA sequence GGGCCC of length 6, which has only four secondary structures, each having $N(s)$ neighbors, given in dot-bracket notation as follows:

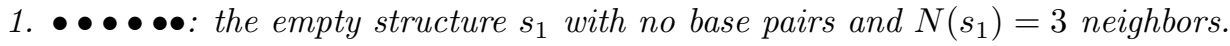

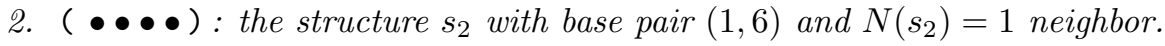

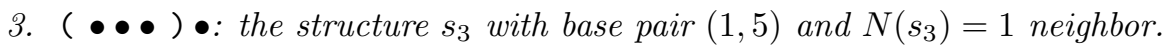

4. $\bullet(\bullet \bullet)$ : the structure $s_{4}$ with base pair $(2,6)$ and $N\left(s_{4}\right)=1$ neighbor.

The transition probability matrix $M$ is given as follows, where we compute the values to 20 decimal places using equation (2), but display only 4 decimal places for notational convenience:

$$
M=\left(\begin{array}{llll}
0.0000 & 0.333 \overline{3} & 0.333 \overline{3} & 0.333 \overline{3} \\
0.1974 & 0.8026 & 0.0000 & 0.0000 \\
0.1974 & 0.8026 & 0.0000 & 0.0000 \\
0.1974 & 0.8026 & 0.0000 & 0.0000
\end{array}\right)
$$

Let $M^{\infty}=\lim _{n \rightarrow \infty} M^{n}$. The stationary probability distribution for $M$ must satisfy $M\left(p_{1}^{*}, p_{2}^{*}, p_{3}^{*}, p_{4}^{*}\right)^{T}=$ $\left(p_{1}^{*}, p_{2}^{*}, p_{3}^{*}, p_{4}^{*}\right)^{T}$, where $T$ denotes transpose, and so

$$
M^{\infty}=\left(\begin{array}{cccc}
p_{1}^{*} & p_{2}^{*} & p_{3}^{*} & p_{4}^{*} \\
p_{1}^{*} & p_{2}^{*} & p_{3}^{*} & p_{4}^{*} \\
p_{1}^{*} & p_{2}^{*} & p_{3}^{*} & p_{4}^{*} \\
p_{1}^{*} & p_{2}^{*} & p_{3}^{*} & p_{4}^{*}
\end{array}\right)
$$

Using the actual 20-place values of the transition probability matrix $M$, whose 4-place approximations are given in equation 21, we obtain the millionth power of $M^{\left(10^{6}\right)}$ by a Mathematica computation. The computed 16-place values are shown to 4-place accuracy in the following:

$$
M^{\left(10^{6}\right)}=\left(\begin{array}{llll}
0.1648 & 0.2784 & 0.2784 & 0.2784 \\
0.1648 & 0.2784 & 0.2784 & 0.2784 \\
0.1648 & 0.2784 & 0.2784 & 0.2784 \\
0.1648 & 0.2784 & 0.2784 & 0.2784
\end{array}\right)
$$


so that the 4-place approximations of the stationary probabilities are as follows: $p_{1}^{*}=0.1648, p_{2}^{*}=0.2784$, $p_{3}^{*}=0.2784, p_{4}^{*}=0.2784$. We then compute that $p_{1}^{*} \cdot p_{1,2}=0.0550$, while $p_{2}^{*} \cdot p_{2,1}=0.0617$, which shows that detailed balance does not hold.

Using the Turner energy model [50, the same example yields a different transition probability matrix (not shown), whose millionth power is as follows:

$M^{\left(10^{6}\right)}=\left(\begin{array}{llll}0.9994655553038468 & 0.000430085352242845 & 0.0000521796443227893 & 0.0000521796443227893 \\ 0.9994655553038468 & 0.000430085352242845 & 0.0000521796443227893 & 0.0000521796443227893 \\ 0.9994655553038468 & 0.000430085352242845 & 0.0000521796443227893 & 0.0000521796443227893 \\ 0.9994655553038468 & 0.000430085352242845 & 0.0000521796443227893 & 0.0000521796443227893\end{array}\right)$

We find that $p_{1}^{*}=0.9994655553038468164 \approx 0.9995, p_{2}^{*}=0.000430315331989701045645180244 \approx 0.0004$, $p_{1,2}=0.001288878388504780024906293256 \approx 0.0013, p_{2,1}=0.061737166286699882156163710079 \approx 0.617$, and that $p_{1}^{*} \cdot p_{1,2}=0.0012881895542860573 \approx 0.00129$ while $p_{2}^{*} \cdot p_{2,1}=0.00002656644920676464 \approx 0.00003$. It follows that detailed balance does not hold for the Turner energy model. For RNA sequences of length $n$, there are exponentially many $\left(\approx 1.95947 \cdot n^{-3 / 2} \cdot 1.86603^{n}\right)$ secondary structures, so similar computations cannot be carried out, and it may be the case that detailed balance "approximately" holds. 